University of Nebraska - Lincoln

DigitalCommons@University of Nebraska - Lincoln

$12-10-1998$

\title{
STEPS TOWARD DETERMINATION OF THE SIZE AND STRUCTURE OF THE BROAD-LINE REGION IN ACTIVE GALACTIC NUCLEI. XIII. ULTRAVIOLET OBSERVATIONS OF THE BROAD-LINE RADIO GALAXY 3C 390.3
}

Paul T. O'Brien

University of Leicester, paul.obrien@leicester.ac.uk

M. Dietrich

Landessternwarte, KoŽ nigstuhl, D-69117 Heidelberg, Germany

K. Leighly

Columbia Astrophysics Laboratory

Poillow this and additional works at: https://digitalcommons, unl.edu/physicsgaskell

Centre dIEtudes de Saclay, Service dïAstrophysique, Orme des Merisiers, 91791 Gif-Sur-Yvette Cedex,

ifurt of the Physics Commons

\section{J. Clavel}

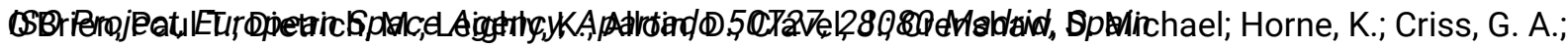
Krolik, Julian Henry; Malkan, Matthew A.; Netzer, Hagai; Peterson, Bradley M.; Reichert, G. A.; Rodriguez-

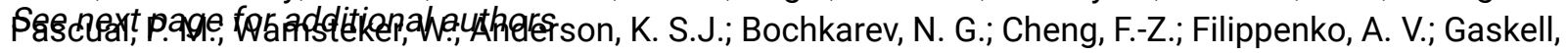
C. Martin; George, I. M.; Goad, M. R.; Ho, L. C.; Kaspi, Shai; Kollatschny, W.; Korista, Kirk T.; MacAlpine, G.; Marlow, D.; Martin, P. G.; Morris, S. L.; Pogge, Richard William; Qian, B. C.; Recondo-Gonzalez, M. C.;

Rodriguez Espinosa, J. M.; Santos-Lleo, M.; Shapovalova, A. I.; Shull, J. M.; Stirpe, G. M.; Sun, W.-H.; Turner, T. J.; Vio, R.; Wagner, S.; Wanders, I.; Wills, K. A.; Wu, H.; Xue, S. J.; and Zou, Z.-L., "STEPS TOWARD DETERMINATION OF THE SIZE AND STRUCTURE OF THE BROAD-LINE REGION IN ACTIVE GALACTIC NUCLEI. XIII. ULTRAVIOLET OBSERVATIONS OF THE BROAD-LINE RADIO GALAXY 3C 390.3" (1998). Martin Gaskell Publications. 9.

https://digitalcommons.unl.edu/physicsgaskell/9

This Article is brought to you for free and open access by the Research Papers in Physics and Astronomy at DigitalCommons@University of Nebraska - Lincoln. It has been accepted for inclusion in Martin Gaskell Publications by an authorized administrator of DigitalCommons@University of Nebraska - Lincoln. 


\section{Authors}

Paul T. O'Brien, M. Dietrich, K. Leighly, D. Alloin, J. Clavel, D. Michael Crenshaw, K. Horne, G. A. Criss, Julian Henry Krolik, Matthew A. Malkan, Hagai Netzer, Bradley M. Peterson, G. A. Reichert, P. M. Rodriguez-

Pascual, W. Wamsteker, K. S.J. Anderson, N. G. Bochkarev, F.-Z. Cheng, A. V. Filippenko, C. Martin Gaskell, I. M. George, M. R. Goad, L. C. Ho, Shai Kaspi, W. Kollatschny, Kirk T. Korista, G. MacAlpine, D. Marlow, P. G. Martin, S. L. Morris, Richard William Pogge, B. C. Qian, M. C. Recondo-Gonzalez, J. M. Rodriguez Espinosa, M. Santos-Lleo, A. I. Shapovalova, J. M. Shull, G. M. Stirpe, W.-H. Sun, T. J. Turner, R. Vio, S. Wagner, I.

Wanders, K. A. Wills, H. Wu, S. J. Xue, and Z.-L. Zou 
The Astrophysical Journal, 509:163-176, 1998 December 10

(C) 1998. The American Astronomical Society. All rights reserved. Printed in U.S.A.

\title{
STEPS TOWARD DETERMINATION OF THE SIZE AND STRUCTURE OF THE BROAD-LINE REGION IN ACTIVE GALACTIC NUCLEI. XIII. ULTRAVIOLET OBSERVATIONS OF THE BROAD-LINE RADIO GALAXY 3C 390.3
}

\author{
P. T. O’Brien, ${ }^{1}$ M. Dietrich, ${ }^{2}$ K. Leighly, ${ }^{3}$ D. Alloin, ${ }^{4}$ J. Clavel ${ }^{5}$ D. M. Crenshaw, ${ }^{6}$ K. Horne, ${ }^{7}$ G. A. Kriss, ${ }^{8}$ \\ J. H. Krolik, ${ }^{8}$ M. A. Malkan, ${ }^{9}$ H. Netzer, ${ }^{10}$ B. M. Peterson, ${ }^{11}$ G. A. Reichert, ${ }^{12}$ P. M. Rodríguez-Pascual, ${ }^{13}$

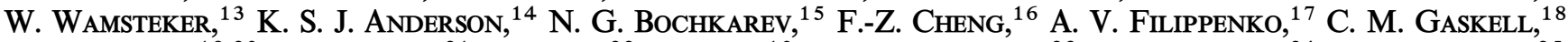 \\ I. M. GeOrge, ${ }^{19,20}$ M. R. GoAd ${ }^{21}$ L. C. Ho, ${ }^{22}$ S. Kaspi, ${ }^{10}$ W. Kollatschny,${ }^{23}$ K. T. Korista, ${ }^{24}$ G. MacAlpine, ${ }^{25}$ \\ D. Marlow, ${ }^{26}$ P. G. Martin, ${ }^{27}$ S. L. Morris, ${ }^{28}$ R. W. Pogge, ${ }^{11}$ B.-C. Qian, ${ }^{29}$ M. C. ReCONDO-Gonzalez, ${ }^{13}$ \\ J. M. Rodríguez Espinosa, ${ }^{13}$ M. Santos-Lleó, ${ }^{30}$ A. I. Shapovalova, ${ }^{31}$ J. M. Shull, ${ }^{32}$ G. M. STIRPe, ${ }^{33}$ \\ W.-H. Sun, ${ }^{34}$ T. J. Turner, ${ }^{19,20}$ R. Vio, ${ }^{13}$ S. WAGner, ${ }^{2}$ I. WANDERS, ${ }^{11,7}$ K. A. Wills, ${ }^{26}$ H. Wu, ${ }^{35}$ \\ S.-J. XUE, ${ }^{16}$ AND Z.-L. ZOU ${ }^{35}$ \\ Received 1997 August 28; accepted 1998 July 14
}

\begin{abstract}
As part of an extensive multiwavelength monitoring campaign, the International Ultraviolet Explorer satellite was used to observe the broad-line radio galaxy 3C 390.3 during the period 1994 December 31-1996 March 5. Spectra were obtained every 6-10 days. The UV continuum varied by a factor of 7 through the campaign, while the broad emission lines varied by factors of 2-5. Unlike previously monitored Seyfert 1 galaxies, in which the X-ray continuum generally varies with a larger amplitude than the UV, in 3C 390.3 the UV continuum light curve is similar in both amplitude and shape to the X-ray light curve observed by ROSAT. The UV broad emission-line variability lags that of the UV continuum by 35-70 days for Ly $\alpha$ and C IV, values larger than those found for Seyfert 1 galaxies of comparable UV luminosity. These lags are also larger than those found for the Balmer lines in 3C 390.3 over the same period. The red and blue wings of C IV and Ly $\alpha$ vary in phase, suggesting that radial motion does not dominate the kinematics of the UV line-emitting gas. Comparison with archival data provides evidence for velocity-dependent changes in the Ly $\alpha$ and C IV line profiles, indicating evolution in the detailed properties and/or distribution of the broad-line emitting gas.
\end{abstract}

Subject headings: galaxies: active — galaxies: individual (3C 390.3) - ultraviolet: galaxies

\footnotetext{
${ }^{1}$ Department of Physics and Astronomy, University of Leicester, University Road, Leicester LE1 7RH, England, UK.

${ }^{2}$ Landessternwarte, Königstuhl, D-69117 Heidelberg, Germany.

${ }^{3}$ Columbia Astrophysics Laboratory, 538 West 120th Street, New York, NY 10027.

${ }^{4}$ Centre d'Etudes de Saclay, Service d'Astrophysique, Orme des Merisiers, 91191 Gif-Sur-Yvette Cedex, France.

${ }^{5}$ ISO Project, European Space Agency, Apartado 50727, 28080 Madrid, Spain.

${ }^{6}$ Computer Sciences Corporation, Laboratory for Astronomy and Solar Physics, NASA Goddard Space Flight Center, Code 681, Greenbelt, MD 20771.

${ }^{7}$ School of Physics and Astronomy, University of Saint Andrews, North Haugh, Saint Andrews KYI 16 9SS, Scotland, UK.

${ }^{8}$ Department of Physics and Astronomy, The Johns Hopkins University, Baltimore, MD 21218.

9 Department of Astronomy, University of California, Math-Science Building, Los Angeles, CA 90024.

${ }^{10}$ School of Physics and Astronomy and the Wise Observatory, The Raymond and Beverly Sackler Faculty of Exact Sciences, Tel-Aviv University, Tel-Aviv 69978, Israel.

${ }^{11}$ Department of Astronomy, The Ohio State University, 174 West 18th Avenue, Columbus, OH 43210.

12 NASA Goddard Space Flight Center, Code 631, Greenbelt, MD 20771.

${ }^{13}$ ESA IUE Observatory, P.O. Box 50727, 28080 Madrid, Spain.

${ }^{14}$ Department of Astronomy, New Mexico State University, Box 30001, Department 4500, Las Cruces, NM 88003.

15 Sternberg Astronomical Institute, M.V. Lomonosov Moscow State University, Universitetskij Prospect, 13, Moscow 119899, Russia.

${ }^{16}$ Center for Astrophysics, University of Science and Technology, Hefei, Anhui, People's Republic, of China.

17 Department of Astronomy, University of California, Berkeley, CA 94720.

18 Department of Physics and Astronomy, University of Nebraska, Lincoln, NE 68588.

${ }^{19}$ Laboratory for High Energy Astrophysics, NASA Goddard Space Flight Center, Greenbelt, MD 20771.

${ }^{20}$ Universities Space Research Association.

${ }^{21}$ Space Telescope Science Institute, 3700 San Martin Drive, Baltimore, MD 21218.

${ }^{22}$ Harvard-Smithsonian Center for Astrophysics, 60 Garden Street, Cambridge, MA 02138.

${ }^{23}$ Universitäts-Sternwarte Göttingen, Geismarlandstrasse 11, D-37083 Göttingen, Germany.

${ }^{24}$ Department of Physics, Western Michigan University, Kalamazoo, MI 49008.

${ }^{25}$ Department of Astronomy, University of Michigan, Dennison Building, Ann Arbor, MI 48109.

${ }^{26}$ Nuffield Radio Astronomy Laboratory, University of Manchester, Jodrell Bank, Macclesfield, Cheshire SK11 9DL, England, UK.

${ }^{27}$ Canadian Institute for Theoretical Astrophysics, University of Toronto, Ontario M5S, 3H8, Canada.

${ }^{28}$ Dominion Astrophysical Observatory, 5071 West Saanich Road, Victoria, British Columbia V8X 4M6, Canada.

${ }^{29}$ Shanghai Astronomical Observatory, Chinese Academy of Sciences, 80 Nandan Lu, Shanghai 20030, China.

${ }^{30}$ LAEFF, Apartado 50727, E-28080 Madrid, Spain.

${ }^{31}$ Special Astrophysical Observatory, Russian Academy of Sciences, Nizhni Arkhiz, Karachaj-Cherkness Region, 357147, Russia.

32 Joint Institute for Laboratory Astrophysics, University of Colorado, and National Institute for Standards and Technology, Campus Box 440, Boulder, CP 80309.

${ }^{33}$ Osservatorio Astronomico di Bologna, Via Zamboni 33, I-40126, Bologna, Italy.

${ }^{34}$ Institute of Astronomy, National Central University, Chung-Li, Taiwan 32054, Republic of China

${ }^{35}$ Beijing Astronomical Observatory, Chinese Academy of Sciences, Beijing 100080, People's Republic of China.
} 


\section{INTRODUCTION}

The broad-line region (BLR) gas in active galactic nuclei (AGN) is believed to be photoionized and heated by the AGN ionizing continuum source, which in turn is generally believed to be powered by the release of gravitational energy when matter is accreted onto a central massive black hole. It is now well established that determining the variability characteristics of AGN provides an invaluable tool with which to investigate their physical nature (see Peterson 1993 and references therein). Indeed, AGN monitoring provides the only practical method at present that can provide spatially resolved information on the nuclear region immediately surrounding the black hole. Broad emission-line variability in response to changes in the ionizing continuum can be used to elucidate the BLR structure through the technique of reverberation mapping (Blandford \& McKee 1982). The multiwaveband continuum variability properties similarly constrain the continuum emission mechanism.

The AGN ionizing continuum can vary significantly on timescales of a few days or less, and the light-crossing time of the BLR may be only a few light-weeks in low-luminosity AGNs. These two factors demand monitoring campaigns with dense sampling patterns, while the unpredictable nature of the continuum variability requires campaigns that typically last several months in order to ensure a high probability of observing variations suitable for measuring the line response. The International AGN Watch was formed to overcome these practical difficulties and to obtain data able to place firm constraints on both the BLR structure and the origin of the luminous AGN continuum. So far, monitoring campaigns have been successfully completed on five Seyfert 1 galaxies: NGC 5548 (Clavel et al. 1991; Peterson et al. 1991, 1992, 1994; Dietrich et al. 1993; Maoz et al. 1993; Korista et al. 1995; Romanishin et al. 1995; Marshall et al. 1997), NGC 3783 (Reichert et al. 1994; Stirpe et al. 1994; Alloin et al. 1995), NGC 4151 (Crenshaw et al. 1996; Kaspi et al. 1996; Warwick et al. 1996; Edelson et al. 1996), Fairall 9 (Rodríguez-Pascual et al. 1997; Santos-Lleó et al. 1997), and NGC 7469 (Wanders et al. 1997; Nandra et al. 1998; Collier et al. 1998).

There are three principal, observational results from the AGN Watch campaigns that appear generic to those Seyfert 1 galaxies intensively monitored: (1) The broad emission lines respond to continuum variations with timedelays (lags) that range from a few days to months. (2) What are commonly referred to as the "high ionization" emission lines (e.g., Ly $\alpha$ and C IV 21549 ) respond on shorter timescales than the "low ionization" lines (e.g., H $\beta$, C III $\lambda 1909$ ), indicating ionization stratification of the BLR. (3) Usually no statistically significant lag has been detected between the optical and UV continuum variations. The only exception to this is NGC 7469, for which Wanders et al. (1997) find a lag of $\sim 0.3$ days between 1315 and $1825 \AA$ using very densely sampled data. For NGC 4151 the lag between the optical and UV continua and between the UV and $1.5 \mathrm{keV}$ $\mathrm{X}$-ray continua are $\leq 1$ and $\leq 0.3$ days, respectively (Edelson et al. 1996). The lack of continuum variability time delays is perhaps the most fundamental result, being difficult to explain using standard accretion disk models in which the variability signal travels radially through the disk. This problem has supported development of thermal reprocessing models in which $\mathrm{X}$-ray photons are reprocessed into UV and optical photons by an accretion disk or
Thomson thick clouds (Guilbert \& Rees 1988; Lightman \& White 1988). Although the details are unclear, these models are capable of explaining at least some of the observations (Krolik et al. 1991; Warwick et al. 1996; Edelson et al. 1996).

The primary reason for the success of these monitoring programs has been the unique capability of the International Ultraviolet Explorer (IUE) to obtain well-calibrated UV spectra at regular, closely spaced intervals. However, despite the past success, it must be stressed that the results summarized above have been obtained only for lowluminosity, radio-quiet AGN. Coordinated UV/X-ray monitoring has also only been possible for relatively short periods. To fully test AGN models, it is essential that multiwavelength data be obtained for a wider range of AGN types over longer temporal baselines. As part of this process, we recently carried out a large X-ray, UV, optical, and radio monitoring campaign on the broad-line radio galaxy (BLRG) 3C 390.3. In this paper we report on the UV campaign. The X-ray, optical, and radio observations are presented elsewhere (Leighly et al. 1997; Dietrich et al. 1998).

The BLRG 3C 390.3 is an ideal target for monitoring. Optical continuum variations of $30 \%-70 \%$ on timescales of weeks to months are common (e.g., Barr et al. 1980), together with large amplitude variations in both the flux and profile of the Balmer emission lines (e.g., Veilleux \& Zheng 1992). Even larger amplitude continuum and broadline variations on similar timescales are seen in the undersampled IUE archival data (Clavel \& Wamsteker 1987; Zheng 1996; Wamsteker et al. 1997). The redshift of 3C $390.3(z=0.056)$ is higher than that of the previously monitored Seyfert 1 galaxies, resulting in a clean separation of the full extent of the broad Ly $\alpha$ plus $N$ v $\lambda 1240$ blend from the geocoronal Ly $\alpha$ line. Of course, the higher redshift is also a disadvantage in that the observed flux is smaller, leading to lower signal-to-noise ratios $(\mathrm{S} / \mathrm{N})$ than in our previous campaigns.

The complex broad-line profiles in 3C 390.3 suggest a highly structured gas distribution and/or anisotropic illumination in the BLR. The Balmer lines have localized peaks that are shifted to substantially higher and lower velocities than the narrow lines. Evidence for such structure is also seen in the C IV $\lambda 1549$ and Ly $\alpha$ lines. This phenomenon of detached line peaks is generally limited to BLRGs. Several explanations have been proposed that the 3C 390.3 line profiles are particularly well suited to test, since they show unusually complex variations. One possibility is a binary black hole system, each with an associated BLR (Gaskell 1988a; Gaskell \& Martin 1996). Eracleous et al. (1997) reject this hypothesis for 3C 390.3, since it implies black hole masses too high to reconcile with the observed spectral and variability properties. An outflowing biconical gas stream has also been proposed (Zheng, Veilleux, \& Grandi 1991), although if an accretion disk is present, as seems essential to fuel the black hole, the far cone would most likely be obscured by the optically thick disk (Livio \& Xu 1997). Emission from an accretion disk (e.g., Perez et al. 1988; Chen, Halpern, \& Filippenko 1989) appears a more likely explanation. Rokaki, Boisson, \& Collin-Souffrin (1992) find an accretion disk inclined at $30^{\circ}$ fits both the overall spectral energy distribution and the broad-line profiles. However, the Balmer line red and blue wings appear to vary independently on long timescales (Zheng et al. 1991), in 
conflict with the simplest disk models. The UV lines also show some evidence for this behavior, although analysis of the same IUE archival data have led to conflicting reports in the literature. Wamsteker et al. (1997) find that the Ly $\alpha$ and $\mathrm{C}$ IV blue wings lag the red, implying radial gas motion, but this result is not confirmed by Zheng (1996). We note that the temporal sampling rate of the archival data is low, with a median sampling interval of 94 days.

3C 390.3 is an edge-brightened radio double (FanaroffRiley type II galaxy), has a relatively strong compact core, and displays superluminal motion (Alef et al. 1996). The latter two properties are usually interpreted as indicating a relativistic jet axis aligned close to the line of sight (Orr \& Browne 1982). It is also a highly variable $X$-ray source, and the $\mathrm{X}$-ray spectrum shows a broad iron $\mathrm{K} \alpha$ line (Inda et al. 1994; Eracleous, Halpern, \& Livio 1996; Leighly et al. 1997). Eracleous et al. (1996) find that an accretion disk inclined at $26^{\circ}$ fits both the radio superluminal data and the X-ray spectra, an inclination angle consistent with that derived by Rokaki et al. (1992). Therefore, in addition to an accretion disk, some of the observed ionizing continuum in 3C 390.3 may arise from a relativistic jet, unlike that of the previously monitored radio-quiet AGN.

Relative to the Seyfert 1 galaxies previously monitored by AGN Watch, 3C 390.3 has a weak "big blue bump." Although the UV luminosity at $1370 \AA, L_{\lambda} \approx(1-2) \times 10^{40}$ ergs s${ }^{-1} \AA^{-1}$, is similar to that of NGC $5548,3 C 390.3$ is a significantly more luminous X-ray source. The weakness of the big blue bump and the historical X-ray light curve led Inda et al. (1994) to suggest that some of the X-ray emission may arise from a similar process to that in radio-loud QSOs, connected with the relativistic jet. Eracleous et al. (1996) note that the X-ray variability in 3C 390.3 does not appear to be related to the superluminal events, although the temporal sampling rate of the published data is too poor to exclude a connection. The monitoring campaign described here was designed to greatly improve on the temporal sampling rates previously achieved so as to place firm constraints on the BLR structure and continuum emission mechanisms in 3C 390.3 .

\section{OBSERVATIONS}

The IUE SWP camera was used to obtain 65 lowdispersion (resolution $\approx 6 \AA$ ) observations of $3 \mathrm{C} 390.3$ at typical intervals of 6-10 days from 1994 December 31 to 1996 March 5. Unfortunately, the IUE campaign had to be curtailed earlier than scheduled because of the loss of a gyro. The observations were all made through the large $\left(10^{\prime \prime} \times 20^{\prime \prime}\right)$ aperture. Because 3C 390.3 is near the ecliptic pole, the high angle between the telescope axis and the antisolar direction (the $\beta$ angle) meant that scattered solar light was present in the telescope tube for most of the observations. This scattered light does not contaminate the extracted short-wavelength prime camera (SWP) spectra (Weinstein \& Carini 1992), but it does raise the background in the Fine Error Sensor (FES) normally used to maintain pointing accuracy during long, continuous exposures. For the epochs when scattered light was present, a number of short-exposure segments, each typically 20 or 30 minutes long, were added together to form each observation. The high $\beta$ angle also required the segmentation of a few exposures to prevent overheating the onboard computer, which could have led to data corruption. For the segmented observations the IUE pointing accuracy was verified between each segment using the FES $X-Y$ position of a nearby bright offset star. This is a standard procedure with IUE (Rodríguez-Pascual et al. 1997), and no evidence was found for any significant guiding errors.

The only real consequences of the scattered light problem are a small loss in on-target exposure time and the absence of an optical flux measurement for the target from the FES. Details of the segmented exposures and FES data can be found in the handwritten logs available at the IUE observatories (observing program IDs SQ037, AGRMM, and RQ079). The SWP observation log is given in Table 1, in which the exposure time is the total integration time from all the segments, while the Julian Date corresponds to the midpoint between the start of the first segment and the end of the last. The UT date and time correspond to the start of the first exposure segment.

\section{DATA REDUCTION AND ANALYSIS}

The IUE spectral images were processed using the NEWSIPS pipeline processing system (Nichols et al. 1993). This software incorporates a new flat-fielding technique and an optimal extraction routine, which together greatly reduce the impact of detector fixed-pattern noise and improve the photometric accuracy. NEWSIPS also incorporates an improved absolute flux calibration based on white dwarf observations. The NEWSIPS output products are FITS files, which contain many quality-control flags in the header and comments from the handwritten logs such as details of the exposure segments. The optimal extraction routine removes most cosmic-ray hits automatically. Together with spurious features due to imperfect removal of reseau marks, those few cosmic rays that remained in the extracted spectra (the MXLO files) were interpolated over before measurement.

The quality-control flags of the NEWSIPS extraction routine allow a further check on the location of the spectrum relative to the center of the large aperture in the spatial direction. No spectrum was found to be more than 4 pixels away from the expected location, which is within the acceptable range to prevent significant vignetting (Rodríguez-Pascual et al. 1997). The spectral location in the dispersion direction was similarly checked by crosscorrelating the narrow peaks of the Ly $\alpha$ and C IV $\lambda 1549$ emission lines. The maximum shift found was only 2 pixels, which is also small enough to have caused no significant flux loss. Integer pixel shifts were determined from the cross-correlation and applied to register the spectra before further analysis. The broad-line peaks in the resulting spectra give a redshift of $0.056 \pm 0.001$, in excellent agreement with that derived from the narrow optical lines (Eracleous et al. 1996).

As well as global quality flags, NEWSIPS provides quality flags at each wavelength in the extracted spectra (the $v$ flag spectrum), which are determined by an algorithm based on the percentage of pixels contributing at each wavelength that suffer from a particular problem (Nichols et al. 1993). During the last third of the 3C 390.3 IUE campaign, the systematically higher continuum and emission-line fluxes led to most spectra having a few pixels in the core of $\mathrm{Ly} \alpha$ being flagged as saturated. Where only a few pixels are saturated this is not a significant problem. However, for seven of the spectra listed in Table 1, between five and ten pixels were flagged. Examination of the spectral images and the $v$ flag spectrum and comparison of these 
TABLE 1

OBSERVATION LOG

\begin{tabular}{|c|c|c|c|c|c|}
\hline $\begin{array}{l}\text { SWP Image } \\
\text { Number }\end{array}$ & UT Date & UT Start & $\begin{array}{l}\text { JD Midpoint } \\
(2,440,000+)\end{array}$ & $\begin{array}{l}\text { Exposure Time } \\
\text { (minutes) }\end{array}$ & Note \\
\hline $53236 \ldots \ldots$ & 1994 Dec 31 & 20.7311 & 9718.364 & 410 & \\
\hline $53286 \ldots \ldots$ & 1995 Jan 5 & 19.3281 & 9723.305 & 420 & \\
\hline $53325 \ldots \ldots \ldots$ & 1995 Jan 11 & 16.2631 & 9729.178 & 420 & \\
\hline $53465 \ldots \ldots$. & 1995 Jan 17 & 16.4417 & 9735.185 & 410 & \\
\hline $53651 \ldots \ldots$. & 1995 Jan 24 & 08.9415 & 9741.873 & 315 & \\
\hline $53825 \ldots \ldots$ & $1995 \mathrm{Feb} 4$ & 15.3592 & 9753.140 & 300 & 1 \\
\hline $53890 \ldots \ldots \ldots$ & $1995 \mathrm{Feb} 11$ & 18.1263 & 9760.255 & 265 & \\
\hline $53928 \ldots \ldots$. & $1996 \mathrm{Feb} 16$ & 09.7267 & 9764.905 & 310 & \\
\hline $53950 \ldots \ldots$ & 1995 Feb 22 & 09.2536 & 9770.886 & 355 & \\
\hline $53996 \ldots \ldots$. & $1995 \mathrm{Feb} 28$ & 09.2804 & 9776.887 & 285 & \\
\hline $54049 \ldots \ldots$. & 1995 Mar 6 & 07.3674 & 9782.807 & 343 & \\
\hline $54108 \ldots \ldots$. & 1995 Mar 12 & 15.5560 & 9789.148 & 305 & \\
\hline $54170 \ldots \ldots$ & 1995 Mar 18 & 15.5179 & 9795.146 & 330 & \\
\hline $54217 \ldots \ldots$. & 1995 Mar 24 & 15.5671 & 9801.148 & 340 & \\
\hline $54260 \ldots \ldots$ & 1995 Mar 30 & 15.2450 & 9807.135 & 345 & \\
\hline $54300 \ldots \ldots$. & 1995 Apr 5 & 13.5896 & 9813.066 & 340 & \\
\hline $54343 \ldots \ldots$ & 1995 Apr 9 & 13.6383 & 9817.068 & 335 & \\
\hline $54453 \ldots \ldots$ & 1995 Apr 17 & 13.2365 & 9825.052 & 365 & \\
\hline $54488 \ldots \ldots$. & 1995 Apr 23 & 03.6946 & 9830.654 & 140 & 2 \\
\hline $54489 \ldots \ldots$ & 1995 Apr 23 & 07.3015 & 9830.804 & 155 & 2 \\
\hline $54506 \ldots \ldots$. & 1995 Apr 26 & 05.4819 & 9833.729 & 330 & \\
\hline $54536 \ldots \ldots$ & 1995 Apr 29 & 05.5161 & 9836.729 & 332 & \\
\hline $54605 \ldots \ldots$. & 1995 May 5 & 03.6367 & 9842.651 & 318 & \\
\hline $54655 \ldots \ldots$ & 1995 May 11 & 03.4326 & 9848.643 & 333 & \\
\hline $54691 \ldots \ldots$ & 1995 May 17 & 03.6199 & 9854.650 & 325 & \\
\hline $54737 \ldots \ldots$ & 1995 May 23 & 12.3044 & 9861.013 & 270 & \\
\hline $54789 \ldots \ldots$ & 1995 May 29 & 11.7160 & 9866.988 & 260 & \\
\hline $54857 \ldots \ldots$. & 1995 Jun 4 & 09.3038 & 9872.888 & 360 & \\
\hline $54972 \ldots \ldots$. & 1995 Jun 10 & 09.1664 & 9878.882 & 345 & \\
\hline $55008 \ldots \ldots$. & 1995 Jun 15 & 09.5492 & 9883.897 & 345 & \\
\hline $55058 \ldots \ldots . .$. & 1995 Jun 22 & 09.5122 & 9890.896 & 330 & \\
\hline $55162 \ldots \ldots$. & 1995 Jun 29 & 09.2940 & 9897.888 & 372 & \\
\hline $55193 \ldots \ldots \ldots$ & 1995 Jul 3 & 07.3666 & 9901.807 & 360 & \\
\hline $55236 \ldots \ldots$. & $1995 \mathrm{Jul} 9$ & 22.7790 & 9908.449 & 278 & \\
\hline $55295 \ldots \ldots \ldots$ & $1995 \mathrm{Jul} 15$ & 22.5633 & 9914.440 & 290 & \\
\hline $55339 \ldots \ldots$ & $1995 \mathrm{Jul} 21$ & 22.9281 & 9920.455 & 320 & \\
\hline $55368 \ldots \ldots$. & $1995 \mathrm{Jul} 27$ & 22.7992 & 9926.450 & 337 & \\
\hline $55408 \ldots \ldots \ldots$ & 1995 Aug 2 & 22.1089 & 9932.421 & 250 & \\
\hline $55450 \ldots \ldots$ & 1995 Aug 8 & 21.7576 & 9938.406 & 260 & \\
\hline $55532 \ldots \ldots$ & 1995 Aug 14 & 21.7831 & 9944.407 & 284 & \\
\hline $55635 \ldots \ldots$. & 1995 Aug 21 & 21.6629 & 9951.402 & 315 & \\
\hline $55749 \ldots \ldots$ & 1995 Aug 28 & 21.8886 & 9958.412 & 285 & \\
\hline $55817 \ldots \ldots$. & $1995 \mathrm{Sep} 4$ & 19.5514 & 9965.314 & 305 & \\
\hline $55908 \ldots \ldots$ & 1995 Sep 11 & 14.3917 & 9972.100 & 130 & 2 \\
\hline $55909 \ldots \ldots$ & 1995 Sep 11 & 19.5281 & 9972.313 & 390 & \\
\hline $55959 \ldots \ldots$ & 1995 Sep 18 & 17.3350 & 9979.222 & 641 & \\
\hline $56016 \ldots \ldots$ & 1995 Sep 25 & 17.6154 & 9986.234 & 580 & 3 \\
\hline $56036 \ldots \ldots$ & 1995 Sep 30 & 17.1876 & 9991.216 & 600 & 4 \\
\hline $56059 \ldots \ldots$ & 1995 Oct 7 & 17.9060 & 9998.246 & 480 & 5 \\
\hline $56087 \ldots \ldots$. & 1995 Oct 14 & 17.7842 & 10005.241 & 480 & 6 \\
\hline $56113 \ldots \ldots . .$. & 1995 Oct 21 & 17.4051 & 10012.226 & 480 & 4 \\
\hline $56130 \ldots \ldots$. & 1995 Oct 28 & 17.8189 & 10019.242 & 480 & \\
\hline $56158 \ldots \ldots$ & 1995 Oct 7 & 15.4439 & 10029.144 & 480 & 7 \\
\hline $56210 \ldots \ldots$. & 1995 Oct 11 & 15.5592 & 10039.148 & 480 & 8 \\
\hline $56243 \ldots \ldots$ & 1995 Oct 27 & 15.3947 & 10049.142 & 480 & 4 \\
\hline $56275 \ldots \ldots$ & 1995 Dec 7 & 17.9117 & 10059.246 & 440 & \\
\hline $56304 \ldots \ldots$. & 1995 Dec 16 & 18.0831 & 10068.253 & 360 & 7 \\
\hline $56358 \ldots \ldots$ & 1995 Dec 28 & 13.1900 & 10080.050 & 450 & 7 \\
\hline $56385 \ldots \ldots$. & 1996 Jan 6 & 11.4000 & 10088.975 & 420 & 9 \\
\hline $56582 \ldots \ldots$. & 1996 Jan 16 & 15.9649 & 10099.165 & 480 & 10 \\
\hline $56637 \ldots \ldots$. & 1996 Jan 26 & 15.0621 & 10109.128 & 430 & 4 \\
\hline $56759 \ldots \ldots$ & 1996 Feb 5 & 17.8221 & 10119.243 & 155 & 2 \\
\hline $56810 \ldots \ldots$. & $1996 \mathrm{Feb} 15$ & 09.9738 & 10128.916 & 480 & 4 \\
\hline $56851 \ldots \ldots$ & $1996 \mathrm{Feb} 25$ & 09.0300 & 10138.876 & 443 & 4 \\
\hline $56893 \ldots \ldots$ & 1996 Mar 5 & 08.4969 & 10147.854 & 480 & 9 \\
\hline
\end{tabular}

NotE.-(1) Image excluded because of unusually high background and poor S/N. (2) Image excluded because of underexposure. (3) 3 pixels saturated in Ly $\alpha$. (4) 1 pixel saturated in Ly $\alpha$. (5) 7 pixels saturated in Ly $\alpha$. (6) 5 pixels saturated in Ly $\alpha$. (7) 6 pixels saturated in Ly $\alpha$. (8) 10 pixels saturated in $L y \alpha$. (9) 2 pixels saturated in $L y \alpha$. (10) 8 pixels saturated in $L y \alpha$. 
flagged spectra with adjacent unsaturated spectra strongly suggest that all of the saturation assignments by NEWSIPS for the 3C 390.3 spectra are borderline cases, where the flux is incorrect by small amounts. Therefore these seven spectra were included in the subsequent analysis, but the analysis was repeated excluding them to check whether they significantly influenced the results.

Of the 65 spectra listed in Table 1, five were excluded from the analysis. Of these, four have exposure times less than 160 minutes and are underexposed. The other, SWP53825, has the unfortunate combination of an unusually high background while the object was faint, resulting in a very noisy spectrum. The mean and median sampling intervals for the 60 good SWP spectra used in the subsequent analysis are 7.2 and 6.4 days, respectively.

\subsection{Measurements}

The average spectrum and the root mean square (rms) deviation from the average derived from the 60 good SWP spectra are shown in Figure 1. The narrow-line components are virtually absent from the rms spectrum, which is as expected since they are not predicted to vary significantly over the timescale of this monitoring campaign. The rms spectrum is a measure of the variable component of the UV spectrum and clearly shows that both the broad emission lines and the continuum varied during the campaign, although the amplitude of variability was higher for the continuum.

All continuum and emission-line fluxes were measured in the observer's frame. It is virtually impossible to find completely line-free continuum wave bands in the UV, particularly longward of C IV $\lambda 1549$, because of Fe II blends. The unweighted average continuum flux was determined in two (observed) wave bands: $1340-1400$ and 1800-1910 $\AA$, henceforth referred to as " $F_{\lambda}(1370 \AA)$ " and " $F_{\lambda}(1855 \AA)$," respectively. For three spectra, noted in Table 2, the $F_{\lambda}(1855 \AA)$ wave band was truncated to avoid spurious features in the spectrum. The continuum fluxes are given in Table 2 and plotted in Figure 2.

The emission-line fluxes for each spectrum were determined by integrating the flux above a first-order polynomial

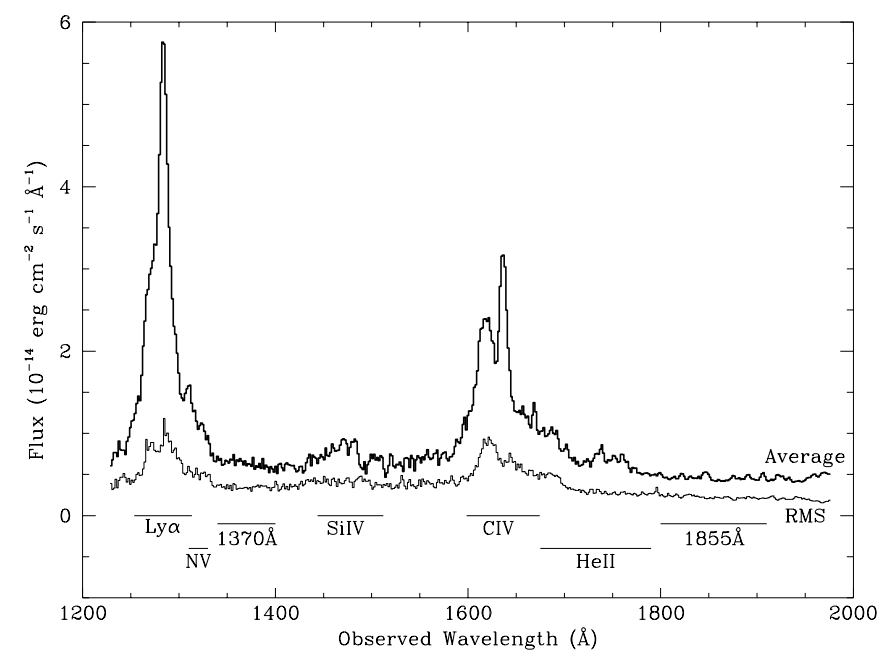

FIG. 1.-Average SWP spectrum of 3C 390.3 (upper thick line) and rms spectrum (lower thin line). The integration limits used for measuring the continuum and major emission-line fluxes are shown below the rms spectrum and are described in the text.
TABLE 2

CONTINUUM FLUXES

\begin{tabular}{|c|c|c|}
\hline \multirow{2}{*}{$\begin{array}{c}\text { JD } \\
(2,440,000+)\end{array}$} & \multicolumn{2}{|c|}{$\begin{array}{c}\text { FLUX } \\
\left(10^{-15} \mathrm{ergs} \mathrm{cm}^{-2} \mathrm{~s}^{-1} \AA^{-1}\right)\end{array}$} \\
\hline & $F_{\lambda}(1370 \AA ̊)$ & $F_{\lambda}(1855 \AA)$ \\
\hline $9718.364 \ldots \ldots$. & $2.76 \pm 0.40$ & $1.18 \pm 0.27$ \\
\hline $9723.306 \ldots \ldots$ & $3.57 \pm 0.62$ & $2.82 \pm 0.31$ \\
\hline $9729.178 \ldots \ldots \ldots$ & $3.21 \pm 0.42$ & $2.17 \pm 0.34$ \\
\hline $9735.186 \ldots$ & $1.86 \pm 0.53$ & $2.19 \pm 0.38$ \\
\hline $9741.873 \ldots$ & $3.30 \pm 0.68$ & $1.24 \pm 0.45$ \\
\hline $9760.255 \ldots \ldots$. & $2.76 \pm 0.53$ & $1.45 \pm 0.47$ \\
\hline $9764.905 \ldots$ & $3.48 \pm 0.84$ & $2.52 \pm 0.89$ \\
\hline $9770.886 \ldots$ & $2.47 \pm 0.37$ & $2.20 \pm 0.41$ \\
\hline $9776.887 .$. & $2.60 \pm 0.42$ & $1.80 \pm 0.50$ \\
\hline 9782.807 .. & $3.75 \pm 0.42$ & $2.35 \pm 0.33$ \\
\hline $9789.148 \ldots \ldots \ldots$ & $4.74 \pm 0.46$ & $2.98 \pm 0.38$ \\
\hline $9795.146 \ldots \ldots$ & $7.44 \pm 0.76$ & $5.17 \pm 0.50$ \\
\hline 9801.148 .. & $5.62 \pm 0.60$ & $3.30 \pm 0.44$ \\
\hline $9807.135 \ldots$ & $4.25 \pm 0.38$ & $3.98 \pm 0.43$ \\
\hline $9813.066 \ldots \ldots \ldots$ & $5.51 \pm 0.29$ & $3.91 \pm 0.31$ \\
\hline $9817.068 \ldots$ & $4.20 \pm 0.59$ & $3.68 \pm 0.50$ \\
\hline $9825.052 \ldots$ & $4.68 \pm 0.38$ & $4.06 \pm 0.39$ \\
\hline 9833.729 . & $5.15 \pm 0.34$ & $3.02 \pm 0.30$ \\
\hline 9836.729 .. & $4.44 \pm 0.59$ & $3.55 \pm 0.57$ \\
\hline $9842.651 \ldots$ & $5.16 \pm 0.59$ & $3.74 \pm 0.46$ \\
\hline $9848.643 \ldots$ & $4.34 \pm 0.61$ & $3.82 \pm 0.50$ \\
\hline $9854.650 \ldots$ & $4.83 \pm 0.37$ & $3.71 \pm 0.32$ \\
\hline $9861.013 \ldots \ldots$. & $5.50 \pm 0.71$ & $4.19 \pm 0.46$ \\
\hline $9866.988 \ldots \ldots \ldots$ & $4.41 \pm 0.62$ & $3.22 \pm 0.51$ \\
\hline $9872.888 \ldots \ldots$. & $4.81 \pm 0.53$ & $3.78 \pm 0.38$ \\
\hline $9878.882 \ldots$ & $5.12 \pm 0.48$ & $4.39 \pm 0.55$ \\
\hline $9883.897 \ldots$ & $4.77 \pm 0.38$ & $3.40 \pm 0.33$ \\
\hline $9890.896 \ldots \ldots \ldots$ & $5.21 \pm 0.45$ & $3.24 \pm 0.36$ \\
\hline $9897.888 \ldots \ldots$. & $5.05 \pm 0.59$ & $3.15 \pm 0.42$ \\
\hline $9901.807 \ldots \ldots$. & $4.04 \pm 0.48$ & $2.71 \pm 0.36$ \\
\hline $9908.449 \ldots \ldots$. & $3.57 \pm 0.58$ & $3.23 \pm 0.44$ \\
\hline $9914.440 \ldots \ldots$ & $3.33 \pm 0.38$ & $3.79 \pm 0.37$ \\
\hline $9920.455 \ldots \ldots \ldots$ & $3.99 \pm 0.55$ & $4.51 \pm 0.43$ \\
\hline $9926.450 \ldots \ldots$ & $4.82 \pm 0.66$ & $4.21 \pm 0.37$ \\
\hline $9932.421 \ldots \ldots$. & $5.10 \pm 0.43$ & $4.31 \pm 0.37$ \\
\hline $9938.406 \ldots \ldots$. & $5.69 \pm 0.58$ & $4.33 \pm 0.48$ \\
\hline $9944.407 \ldots \ldots \ldots$ & $6.64 \pm 0.72$ & $5.80 \pm 0.80$ \\
\hline $9951.402 \ldots \ldots$. & $7.73 \pm 0.77$ & $6.64 \pm 0.57^{\mathrm{a}}$ \\
\hline $9958.412 \ldots \ldots$. & $7.35 \pm 0.51$ & $5.11 \pm 0.45$ \\
\hline $9965.314 \ldots \ldots$. & $6.49 \pm 0.60$ & $5.55 \pm 0.45$ \\
\hline $9972.313 \ldots \ldots$. & $7.16 \pm 0.45$ & $5.27 \pm 0.30$ \\
\hline $9979.223 \ldots \ldots \ldots$ & $5.29 \pm 0.50$ & $4.20 \pm 0.39$ \\
\hline $9986.234 \ldots \ldots$. & $8.51 \pm 0.49$ & $5.70 \pm 0.33$ \\
\hline $9991.216 \ldots \ldots \ldots$ & $7.18 \pm 0.41$ & $5.32 \pm 0.76$ \\
\hline $9998.246 \ldots \ldots$. & $8.02 \pm 1.02$ & $5.34 \pm 0.54$ \\
\hline $10005.241 \ldots \ldots$ & $9.48 \pm 1.24$ & $5.38 \pm 0.42$ \\
\hline $10012.226 \ldots \ldots$ & $10.15 \pm 0.51$ & $6.48 \pm 0.38$ \\
\hline $10019.242 \ldots \ldots$ & $10.51 \pm 0.31$ & $7.42 \pm 0.33$ \\
\hline $10029.144 \ldots \ldots$ & $13.56 \pm 0.47$ & $8.18 \pm 0.45$ \\
\hline $10039.148 \ldots \ldots$ & $13.63 \pm 0.68$ & $8.99 \pm 0.45^{b}$ \\
\hline $10049.142 \ldots \ldots$ & $12.95 \pm 0.45$ & $7.44 \pm 0.31$ \\
\hline $10059.246 \ldots \ldots$ & $9.37 \pm 0.48$ & $6.37 \pm 0.38$ \\
\hline $10068.253 \ldots \ldots$ & $9.43 \pm 0.44$ & $7.52 \pm 0.50$ \\
\hline $10080.050 \ldots \ldots$ & $9.32 \pm 0.59$ & $6.37 \pm 0.56$ \\
\hline $10088.975 \ldots \ldots$ & $8.91 \pm 0.55$ & $6.88 \pm 0.39$ \\
\hline $10099.165 \ldots \ldots$ & $8.49 \pm 0.61$ & $6.19 \pm 0.40$ \\
\hline $10109.128 \ldots \ldots$ & $9.36 \pm 0.55$ & $7.32 \pm 0.40$ \\
\hline $10128.916 \ldots \ldots$ & $10.84 \pm 0.53$ & $7.36 \pm 0.37$ \\
\hline $10138.876 \ldots \ldots$ & $12.03 \pm 0.42$ & $7.67 \pm 0.27$ \\
\hline $10147.854 \ldots \ldots$ & $12.05 \pm 0.51$ & $8.67 \pm 0.35^{\mathrm{a}}$ \\
\hline
\end{tabular}

${ }^{\text {a }}$ Wave band truncated to $1800-1840 \AA$.

b Wave band truncated to $1800-1870 \AA$.

fitted to the individual data within the continuum wave bands. For each line, fixed (observed) wavelength limits were used for the integration. For Ly $\alpha$ and $C$ IV $\lambda 1549$, these limits were $1253.5-1313.5 \AA$ and $1598.5-1674.5 \AA$, respectively, corresponding to a velocity range of $\pm 7000 \mathrm{~km} \mathrm{~s}^{-1}$ 


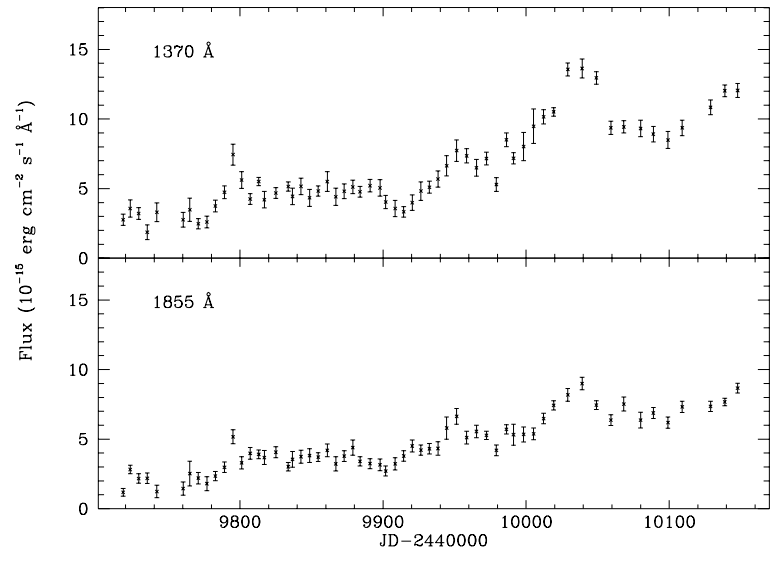

FIG. 2.-Continuum light curves

relative to the (observed) line peaks at 1283.5 and $1636.5 \AA$. These velocity limits correspond to those used by Wamsteker et al. (1997). The Ly $\alpha$ and C IV $\lambda 1549$ red wings will contain contributions from $\mathrm{N} v \lambda 1240$ and $\mathrm{He}$ II $\lambda 1640$ (see below), but no attempt was made to deblend these features. The total (blended) line fluxes will henceforth be referred to as $F(\mathrm{Ly} \alpha)$ and $F(\mathrm{C}$ IV). To search for velocitydependent variations in line flux, the Ly $\alpha$ and $C$ IV $\lambda 1549$ lines were also measured using three components-red, center, and blue. The center corresponds to $\pm 2000 \mathrm{~km} \mathrm{~s}^{-1}$ while the red and blue are $\pm 2000-7000 \mathrm{~km} \mathrm{~s}^{-1}$, respectively. The Ly $\alpha$ and $C$ Iv $\lambda 1549$ fluxes are given in Table 3 and plotted in Figure 3.

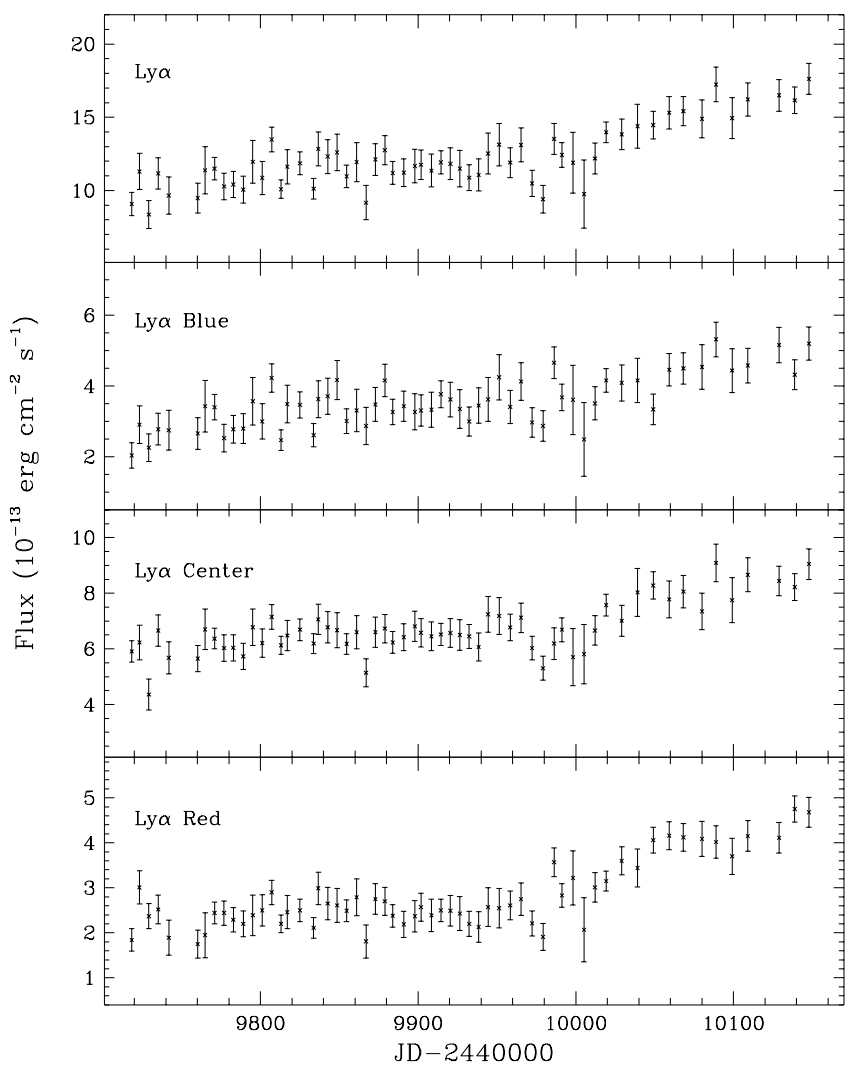

FIG. $3 a$
Determination of the emission-line fluxes for the weaker $\mathrm{N} v \lambda 1240$, Si IV $\lambda 1400$, and He II $\lambda 1640$ lines is more difficult because of the mediocre spectral quality. Based on the rms spectra, the $\mathrm{N} \vee \lambda 1240$ flux was measured in the range $1310-1330 \AA$. This overlaps with the Ly $\alpha$ measurement and only covers the red wing of $N$ v $\lambda 1240$ to approximately $+5000 \mathrm{~km} \mathrm{~s}^{-1}$. For Si IV $\lambda 1400$, limits of $1444-1512 \AA$ were used corresponding to $\pm 7000 \mathrm{~km} \mathrm{~s}^{-1}$. For He II $\lambda 1640$, the rms spectrum shows little evidence for variability, so the flux was measured using limits of 1675-1790 $\AA( \pm 10,000$ $\mathrm{km} \mathrm{s}^{-1}$ ). The $\mathrm{N} v \lambda 1240$, Si Iv $\lambda 1400$, and He II $\lambda 1640$ fluxes are given in Table 4 and plotted in Figure 4.

An additional " $\mathrm{C}$ IV (far-red)" flux was measured in the range $1670-1697 \AA$ to study the possible fast variability of a "shelf" of emission seen in the rms spectrum (§ 3.3.2) centered at $\approx 8500 \mathrm{~km} \mathrm{~s}^{-1}$. This feature is more prominent than in the rms spectrum calculated by Wamsteker et al. (1997) from the IUE archival data. A similar feature is also seen in the Balmer lines during 1994-1995 (Dietrich et al. 1998).

The total and center emission-line fluxes include contributions from the narrow-line region and are uncorrected for absorption either intrinsic to 3C 390.3 or due to our Galaxy. Spectra taken with the Faint Object Spectrograph on the Hubble Space Telescope (Eracleous et al. 1998) suggest that the narrow-line region contributes $\sim 20 \%$ of the mean flux of the center component for Ly $\alpha$ and C IV $\lambda 1549$.

To estimate the uncertainties on the continuum and emission-line fluxes we adopted the following procedure: First, "observed" flux uncertainties were assigned at each epoch based on the spectral data contributing to the contin-

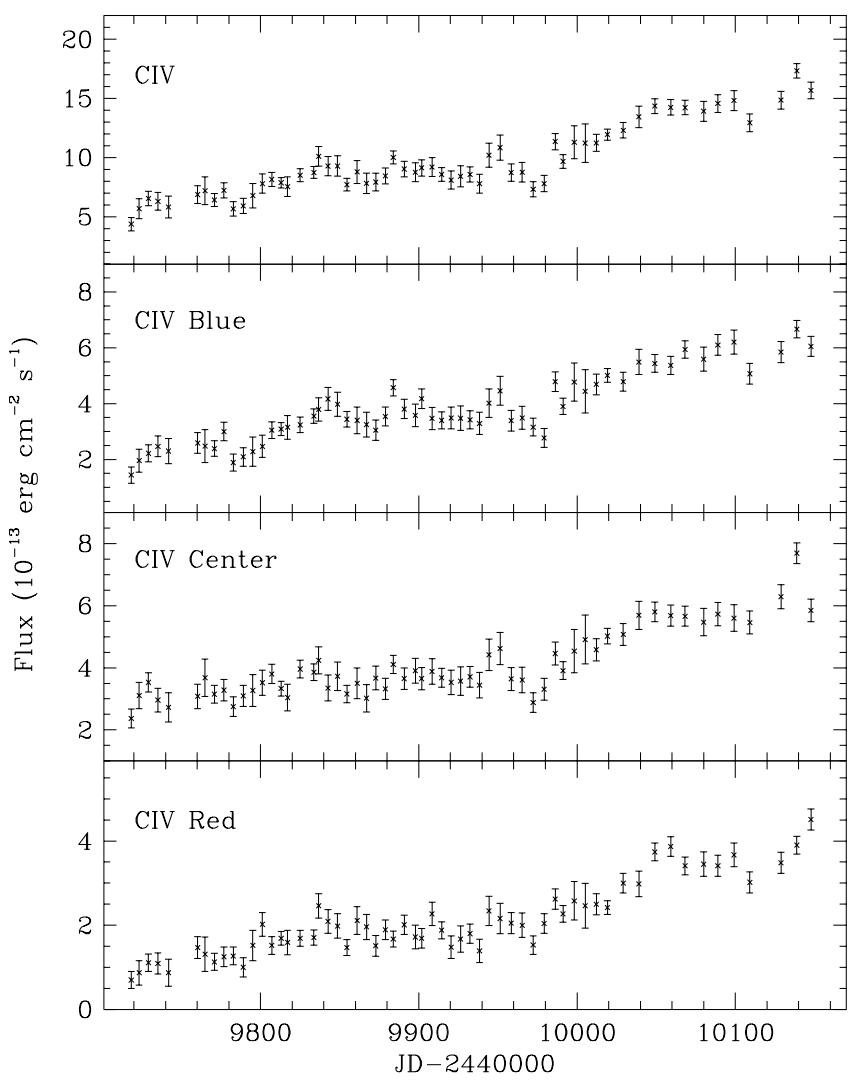

FIG. $3 b$

Fig. 3. - Light curves for (a) Ly $\alpha$ and (b) C Iv $\lambda 1549$ 
TABLE 3

EMISSION-LiNE FLUXES FOR LY $\alpha$ AND C IV $\lambda 1549$

\begin{tabular}{|c|c|c|c|c|c|c|c|c|}
\hline \multirow{2}{*}{$\begin{array}{c}\text { JD } \\
(2,440,000+)\end{array}$} & \multicolumn{4}{|c|}{$\begin{array}{c}\mathrm{Ly} \alpha \\
\left(10^{-13} \mathrm{ergs} \mathrm{cm}^{-2} \mathrm{~s}^{-1}\right)\end{array}$} & \multicolumn{4}{|c|}{$\begin{array}{c}\text { C IV } \lambda 1549 \\
\left(10^{-13} \mathrm{ergs}^{-2} \mathrm{sm}^{-1}\right)\end{array}$} \\
\hline & Total & Blue & Center & Red & Total & Blue & Center & Red \\
\hline 9718.364 . & $9.08 \pm 0.80$ & $2.04 \pm 0.36$ & $5.91 \pm 0.39$ & $1.84 \pm 0.25$ & $4.37 \pm 0.57$ & $1.44 \pm 0.29$ & $2.37 \pm 0.30$ & $0.70 \pm 0.20$ \\
\hline 9723.306 . & $11.30 \pm 1.23$ & $.91 \pm 0.53$ & $6.23 \pm 0.62$ & $3.01 \pm 0.37$ & $5.68 \pm 0.84$ & $1.96 \pm 0.41$ & $3.11 \pm 0.42$ & $0.87 \pm 0.29$ \\
\hline 9729.178 . & $8.36 \pm 0.95$ & $2.26 \pm 0.39$ & $4.36 \pm 0.56$ & $2.37 \pm 0.28$ & $6.54 \pm 0.60$ & $2.22 \pm 0.30$ & $3.53 \pm 0.31$ & $1.11 \pm 0.21$ \\
\hline 9735.186 . & $11.17 \pm 1.07$ & $2.78 \pm 0.45$ & $6.66 \pm 0.56$ & $2.52 \pm 0.32$ & $6.30 \pm 0.74$ & $2.47 \pm 0.37$ & $2.96 \pm 0.38$ & $1.09 \pm 0.25$ \\
\hline 9741.873 . & $9.66 \pm 1.27$ & $2.75 \pm 0.56$ & $5.68 \pm 0.58$ & $1.89 \pm 0.39$ & $5.82 \pm 0.92$ & $2.30 \pm 0.45$ & $2.73 \pm 0.47$ & $0.87 \pm 0.32$ \\
\hline 9760.255 . & $9.48 \pm 1.01$ & $2.66 \pm 0.45$ & $5.65 \pm 0.47$ & $1.75 \pm 0.31$ & $6.87 \pm 0.74$ & $2.59 \pm 0.37$ & $3.08 \pm 0.39$ & $1.47 \pm 0.26$ \\
\hline $9764.905 \ldots$ & $11.38 \pm 1.61$ & $3.43 \pm 0.73$ & $6.70 \pm 0.73$ & $1.95 \pm 0.50$ & $7.20 \pm 1.17$ & $2.48 \pm 0.59$ & $3.68 \pm 0.60$ & $1.31 \pm 0.41$ \\
\hline $9770.886 \ldots$ & $11.50 \pm 0.76$ & $3.40 \pm 0.36$ & $6.37 \pm 0.37$ & $2.44 \pm 0.24$ & $6.41 \pm 0.55$ & $2.39 \pm 0.28$ & $3.15 \pm 0.29$ & $1.13 \pm 0.20$ \\
\hline $9776.887 \ldots$ & $10.28 \pm 0.90$ & $2.53 \pm 0.39$ & $6.03 \pm 0.48$ & $2.44 \pm 0.27$ & $7.22 \pm 0.64$ & $3.00 \pm 0.33$ & $3.28 \pm 0.35$ & $1.25 \pm 0.23$ \\
\hline 9782.807 . & $10.41 \pm 0.89$ & $2.78 \pm 0.39$ & $6.04 \pm 0.47$ & $2.29 \pm 0.27$ & $5.67 \pm 0.61$ & $1.89 \pm 0.31$ & $2.75 \pm 0.32$ & $1.27 \pm 0.21$ \\
\hline 9789.148 . & $10.06 \pm 0.93$ & $2.80 \pm 0.42$ & $5.73 \pm 0.47$ & $2.20 \pm 0.29$ & $5.92 \pm 0.65$ & $2.09 \pm 0.33$ & $3.10 \pm 0.34$ & $1.00 \pm 0.23$ \\
\hline $9795.146 \ldots$ & $11.96 \pm 1.46$ & $3.57 \pm 0.67$ & $6.78 \pm 0.66$ & $2.39 \pm 0.45$ & $6.78 \pm 1.03$ & $2.28 \pm 0.52$ & $3.27 \pm 0.51$ & $1.52 \pm 0.36$ \\
\hline $9801.148 \ldots$ & $10.86 \pm 1.13$ & $3.00 \pm 0.50$ & $6.21 \pm 0.51$ & $2.50 \pm 0.35$ & $7.80 \pm 0.82$ & $2.47 \pm 0.40$ & $3.52 \pm 0.41$ & $2.02 \pm 0.28$ \\
\hline 9807.135 . & $13.49 \pm 0.84$ & $4.23 \pm 0.40$ & $7.15 \pm 0.44$ & $2.90 \pm 0.27$ & $8.15 \pm 0.58$ & $3.05 \pm 0.30$ & $3.80 \pm 0.31$ & $1.52 \pm 0.21$ \\
\hline 9813.066 . & $10.09 \pm 0.63$ & $2.47 \pm 0.29$ & $6.13 \pm 0.32$ & $2.20 \pm 0.20$ & $7.87 \pm 0.44$ & $3.09 \pm 0.23$ & $3.33 \pm 0.24$ & $1.69 \pm 0.16$ \\
\hline 9817.068 . & $11.63 \pm 1.17$ & $3.49 \pm 0.53$ & $6.48 \pm 0.54$ & $2.46 \pm 0.37$ & $7.53 \pm 0.83$ & $3.15 \pm 0.42$ & $3.04 \pm 0.43$ & $1.59 \pm 0.29$ \\
\hline $9825.052 \ldots \ldots$ & $11.86 \pm 0.79$ & $3.47 \pm 0.37$ & $6.69 \pm 0.39$ & $2.50 \pm 0.25$ & $8.51 \pm 0.55$ & $3.24 \pm 0.28$ & $3.96 \pm 0.29$ & $1.69 \pm 0.19$ \\
\hline 9833.729 . & $10.12 \pm 0.71$ & $2.61 \pm 0.33$ & $6.19 \pm 0.36$ & $2.11 \pm 0.23$ & $8.73 \pm 0.50$ & $3.55 \pm 0.26$ & $3.86 \pm 0.27$ & $1.71 \pm 0.18$ \\
\hline 9836.729 . & $12.84 \pm 1.15$ & $3.63 \pm 0.52$ & $7.06 \pm 0.54$ & $2.99 \pm 0.36$ & $10.10 \pm 0.83$ & $3.79 \pm 0.42$ & $4.24 \pm 0.43$ & $2.46 \pm 0.29$ \\
\hline $9842.651 \ldots$ & $12.32 \pm 1.16$ & $3.71 \pm 0.51$ & $6.78 \pm 0.56$ & $2.65 \pm 0.36$ & $9.28 \pm 0.81$ & $4.17 \pm 0.41$ & $3.35 \pm 0.42$ & $2.09 \pm 0.28$ \\
\hline $9848.643 \ldots \ldots$ & $12.60 \pm 1.24$ & $4.17 \pm 0.55$ & $6.67 \pm 0.63$ & $2.61 \pm 0.38$ & $9.28 \pm 0.86$ & $3.98 \pm 0.43$ & $3.73 \pm 0.46$ & $1.98 \pm 0.29$ \\
\hline $9854.650 \ldots$ & $10.97 \pm 0.76$ & $3.01 \pm 0.35$ & $6.18 \pm 0.37$ & $2.49 \pm 0.24$ & $7.71 \pm 0.54$ & $3.44 \pm 0.28$ & $3.16 \pm 0.28$ & $1.47 \pm 0.19$ \\
\hline $9861.013 \ldots$ & $11.94 \pm 1.34$ & $3.31 \pm 0.60$ & $6.60 \pm 0.60$ & $2.79 \pm 0.41$ & $8.78 \pm 0.97$ & $3.40 \pm 0.48$ & $3.50 \pm 0.49$ & $2.11 \pm 0.33$ \\
\hline $9866.988 \ldots$ & $9.17 \pm 1.17$ & $2.87 \pm 0.53$ & $5.14 \pm 0.51$ & $1.81 \pm 0.37$ & $7.82 \pm 0.87$ & $3.25 \pm 0.44$ & $3.02 \pm 0.44$ & $1.96 \pm 0.30$ \\
\hline $9872.888 \ldots$. & $12.12 \pm 1.08$ & $3.48 \pm 0.48$ & $6.60 \pm 0.54$ & $2.75 \pm 0.34$ & $7.93 \pm 0.74$ & $3.05 \pm 0.37$ & $3.67 \pm 0.38$ & $1.51 \pm 0.25$ \\
\hline $9878.882 \ldots \ldots$ & $12.75 \pm 1.00$ & $4.16 \pm 0.46$ & $6.73 \pm 0.51$ & $2.70 \pm 0.31$ & $8.44 \pm 0.67$ & $3.54 \pm 0.34$ & $3.32 \pm 0.34$ & $1.89 \pm 0.23$ \\
\hline $9883.897 \ldots \ldots$ & $11.20 \pm 0.78$ & $3.27 \pm 0.36$ & $6.23 \pm 0.39$ & $2.38 \pm 0.25$ & $10.01 \pm 0.56$ & $4.57 \pm 0.29$ & $4.11 \pm 0.29$ & $1.67 \pm 0$ \\
\hline $9890.896 \ldots \ldots$ & $11.22 \pm 0.94$ & $3.43 \pm 0.43$ & $6.42 \pm 0.48$ & $2.19 \pm 0.29$ & $9.03 \pm 0.66$ & $3.81 \pm 0.34$ & $3.65 \pm 0.35$ & $2.01 \pm 0.23$ \\
\hline $9897.888 \ldots \ldots$ & $11.67 \pm 1.15$ & $3.27 \pm 0.51$ & $6.81 \pm 0.54$ & $2.37 \pm 0.35$ & $8.76 \pm 0.81$ & $3.58 \pm 0.40$ & $3.91 \pm 0.40$ & $1.72 \pm 0.28$ \\
\hline $9901.807 \ldots .$. & $11.76 \pm 1.00$ & $3.31 \pm 0.44$ & $6.58 \pm 0.51$ & $2.57 \pm 0.31$ & $9.13 \pm 0.69$ & $4.18 \pm 0.35$ & $3.65 \pm 0.36$ & $1.69 \pm 0.23$ \\
\hline $9908.449 \ldots \ldots$ & $11.36 \pm 1.12$ & $3.33 \pm 0.50$ & $6.45 \pm 0.52$ & $2.39 \pm 0.36$ & $9.20 \pm 0.81$ & $3.47 \pm 0.40$ & $3.88 \pm 0.41$ & $2.27 \pm 0$ \\
\hline $9914.440 \ldots \ldots$. & $11.93 \pm 0.80$ & $3.77 \pm 0.38$ & $6.52 \pm 0.40$ & $2.50 \pm 0.25$ & $8.58 \pm 0.57$ & $3.40 \pm 0.30$ & $3.68 \pm 0.30$ & $1.88 \pm 0.20$ \\
\hline $9920.455 \ldots \ldots$ & $11.83 \pm 1.08$ & $3.62 \pm 0.49$ & $6.57 \pm 0.52$ & $2.49 \pm 0.34$ & $8.09 \pm 0.77$ & $3.49 \pm 0.39$ & $3.53 \pm 0.39$ & $1.48 \pm 0.27$ \\
\hline $9926.450 \ldots \ldots$. & $11.50 \pm 1.24$ & $3.35 \pm 0.55$ & $6.50 \pm 0.55$ & $2.43 \pm 0.38$ & $8.41 \pm 0.90$ & $3.48 \pm 0.44$ & $3.57 \pm 0.46$ & $1.67 \pm 0.31$ \\
\hline $9932.421 \ldots \ldots$. & $10.88 \pm 0.88$ & $3.00 \pm 0.41$ & $6.45 \pm 0.43$ & $2.20 \pm 0.28$ & $8.58 \pm 0.64$ & $3.42 \pm 0.33$ & $3.71 \pm 0.33$ & $1.80 \pm 0.23$ \\
\hline 9938.406. & $11.06 \pm 1.10$ & $3.45 \pm 0.50$ & $6.07 \pm 0.50$ & $2.13 \pm 0.34$ & $7.79 \pm 0.80$ & $3.29 \pm 0.40$ & $3.44 \pm 0.41$ & $1.39 \pm 0.28$ \\
\hline $9944.407 \ldots \ldots$. & $12.53 \pm 1.39$ & $3.62 \pm 0.62$ & $7.24 \pm 0.64$ & $2.57 \pm 0.43$ & $10.20 \pm 1.01$ & $4.02 \pm 0.51$ & $4.42 \pm 0.51$ & $2.34 \pm 0.35$ \\
\hline $9951.402 \ldots \ldots$ & $13.13 \pm 1.45$ & $4.25 \pm 0.64$ & $7.18 \pm 0.66$ & $2.55 \pm 0.44$ & $10.84 \pm 1.05$ & $4.46 \pm 0.52$ & $4.62 \pm 0.52$ & $2.16 \pm 0.36$ \\
\hline $9958.412 \ldots \ldots$ & $11.91 \pm 1.02$ & $3.41 \pm 0.47$ & $6.77 \pm 0.48$ & $2.61 \pm 0.32$ & $8.73 \pm 0.72$ & $3.39 \pm 0.37$ & $3.64 \pm 0.37$ & $2.05 \pm 0.25$ \\
\hline $9965.314 \ldots \ldots$ & $13.11 \pm 1.16$ & $4.13 \pm 0.53$ & $7.12 \pm 0.53$ & $2.75 \pm 0.36$ & $8.77 \pm 0.83$ & $3.49 \pm 0.41$ & $3.61 \pm 0.41$ & $2.00 \pm 0.29$ \\
\hline $9972.313 \ldots \ldots$ & $10.49 \pm 0.90$ & $2.97 \pm 0.42$ & $6.03 \pm 0.43$ & $2.21 \pm 0.28$ & $7.32 \pm 0.63$ & $3.16 \pm 0.32$ & $2.88 \pm 0.32$ & $1.53 \pm 0.22$ \\
\hline $9979.223 \ldots \ldots$. & $9.41 \pm 0.95$ & $2.87 \pm 0.43$ & $5.30 \pm 0.43$ & $1.91 \pm 0.30$ & $7.80 \pm 0.68$ & $2.77 \pm 0.34$ & $3.31 \pm 0.35$ & $2.04 \pm 0.23$ \\
\hline $9986.234 \ldots \ldots$ & $13.52 \pm 1.05$ & $4.66 \pm 0.45$ & $6.19 \pm 0.57$ & $3.57 \pm 0.32$ & $11.35 \pm 0.69$ & $4.79 \pm 0.35$ & $4.46 \pm 0.37$ & $2.62 \pm 0.24$ \\
\hline $9991.216 \ldots \ldots$. & $12.43 \pm 0.85$ & $3.68 \pm 0.38$ & $6.69 \pm 0.43$ & $2.83 \pm 0.26$ & & $3.90 \pm 0.29$ & $3.91 \pm 0.29$ & $2.27 \pm 0.20$ \\
\hline $9998.246 \ldots \ldots$ & $11.90 \pm 2.08$ & $3.61 \pm 0.98$ & $5.70 \pm 1.03$ & $3.22 \pm 0.60$ & $11.29 \pm 1.38$ & $4.77 \pm 0.68$ & $4.54 \pm 0.70$ & $2.58 \pm 0.46$ \\
\hline $10005.241 \ldots \ldots$ & $9.76 \pm 2.34$ & $2.49 \pm 1.04$ & $5.81 \pm 1.07$ & $2.07 \pm 0.71$ & $11.22 \pm 1.62$ & $4.44 \pm 0.78$ & $4.91 \pm 0.79$ & $2.46 \pm 0.53$ \\
\hline $10012.226 \ldots \ldots$ & $12.19 \pm 1.05$ & $3.51 \pm 0.47$ & $6.66 \pm 0.53$ & $3.01 \pm 0.33$ & $11.24 \pm 0.72$ & $4.69 \pm 0.37$ & $4.58 \pm 0.36$ & $2.50 \pm 0.25$ \\
\hline $10019.242 \ldots \ldots$ & $13.98 \pm 0.71$ & $4.16 \pm 0.33$ & $7.57 \pm 0.39$ & $3.15 \pm 0.22$ & $11.94 \pm 0.46$ & $5.01 \pm 0.24$ & $5.02 \pm 0.25$ & $2.42 \pm 0.16$ \\
\hline $10029.144 \ldots \ldots$ & $13.83 \pm 1.04$ & $4.09 \pm 0.51$ & $7.01 \pm 0.55$ & $3.60 \pm 0.31$ & & $4.79 \pm 0.34$ & $5.07 \pm 0.35$ & $3.00 \pm 0.23$ \\
\hline $10039.148 \ldots \ldots$ & $14.39 \pm 1.49$ & $4.16 \pm 0.63$ & $8.03 \pm 0.86$ & $3.44 \pm 0.42$ & $13.44 \pm 0.91$ & $5.49 \pm 0.45$ & $5.69 \pm 0.45$ & $2.98 \pm 0.30$ \\
\hline $10049.142 \ldots \ldots$ & $14.46 \pm 0.95$ & $3.34 \pm 0.43$ & $8.28 \pm 0.49$ & $4.06 \pm 0.29$ & $14.35 \pm 0.63$ & $5.44 \pm 0.32$ & $5.80 \pm 0.32$ & $3.74 \pm 0.21$ \\
\hline $10059.246 \ldots \ldots$ & $15.31 \pm 1.10$ & $4.46 \pm 0.46$ & $7.78 \pm 0.66$ & $4.16 \pm 0.31$ & $14.24 \pm 0.66$ & $5.37 \pm 0.33$ & $5.68 \pm 0.34$ & $3.87 \pm 0.23$ \\
\hline $10068.253 \ldots \ldots$ & $15.42 \pm 1.00$ & $4.50 \pm 0.44$ & $8.06 \pm 0.58$ & $4.12 \pm 0.31$ & $14.22 \pm 0.61$ & $5.94 \pm 0.31$ & $5.66 \pm 0.32$ & $3.41 \pm 0.21$ \\
\hline $10080.050 \ldots \ldots$ & $14.89 \pm 1.29$ & $4.54 \pm 0.63$ & $7.35 \pm 0.66$ & $4.09 \pm 0.39$ & $13.91 \pm 0.84$ & $5.59 \pm 0.43$ & $5.47 \pm 0.44$ & $3.45 \pm 0.29$ \\
\hline $10088.975 \ldots \ldots$ & $17.24 \pm 1.18$ & $5.32 \pm 0.49$ & $9.09 \pm 0.67$ & $4.02 \pm 0.36$ & $14.57 \pm 0.75$ & $6.10 \pm 0.37$ & $5.73 \pm 0.38$ & $3.41 \pm 0.25$ \\
\hline $10099.165 \ldots \ldots$ & $14.94 \pm 1.39$ & $4.44 \pm 0.62$ & $7.75 \pm 0.81$ & $3.70 \pm 0.40$ & $14.82 \pm 0.84$ & $6.20 \pm 0.43$ & $5.60 \pm 0.43$ & $3.67 \pm 0.28$ \\
\hline $10109.128 \ldots \ldots$ & $16.22 \pm 1.14$ & $4.58 \pm 0.49$ & $8.66 \pm 0.61$ & $4.15 \pm 0.34$ & $12.94 \pm 0.75$ & $5.07 \pm 0.37$ & $5.46 \pm 0.37$ & $3.02 \pm 0.25$ \\
\hline $10128.916 \ldots \ldots$ & $16.50 \pm 1.08$ & $5.16 \pm 0.50$ & $8.44 \pm 0.53$ & $4.11 \pm 0.34$ & $14.85 \pm 0.75$ & $5.85 \pm 0.38$ & $6.29 \pm 0.39$ & $3.48 \pm 0.25$ \\
\hline & $16.16 \pm 0.91$ & $4.32 \pm 0.42$ & $8.22 \pm 0.48$ & $4.75 \pm 0.29$ & $17.34 \pm 0.61$ & $6.67 \pm 0.31$ & $7.69 \pm 0.33$ & $3.90 \pm 0.21$ \\
\hline $10147.854 \ldots \ldots$ & $17.62 \pm 1.06$ & $5.20 \pm 0.47$ & $9.05 \pm 0.55$ & $4.68 \pm 0.33$ & $15.68 \pm 0.71$ & $6.05 \pm 0.36$ & $5.85 \pm 0.36$ & $4.51 \pm 0.25$ \\
\hline
\end{tabular}

uum and emission-line fluxes. We then assume there is no intrinsic variability between consecutive epochs $i, j$ within some monitoring period. The rms of the distribution of flux differences $\left(F_{j}-F_{i}\right)$ for all the epochs within the monitoring period is then calculated and compared to the mean observed uncertainty. This provides a scale factor with which to rescale all of the observed uncertainties so that the rms of the flux differences is consistent with that expected from the mean (rescaled) flux uncertainty.

Rodríguez-Pascual et al. (1997) and Wanders et al. (1997) used an analogous procedure to calculate the flux uncertainties for Fairall 9 and NGC 7469, based on the rms of the distribution of flux ratios $\left(F_{j} / F_{i}\right)$ rather than flux differences. Applied to the 3C 390.3 data, their procedure gives rescaled 


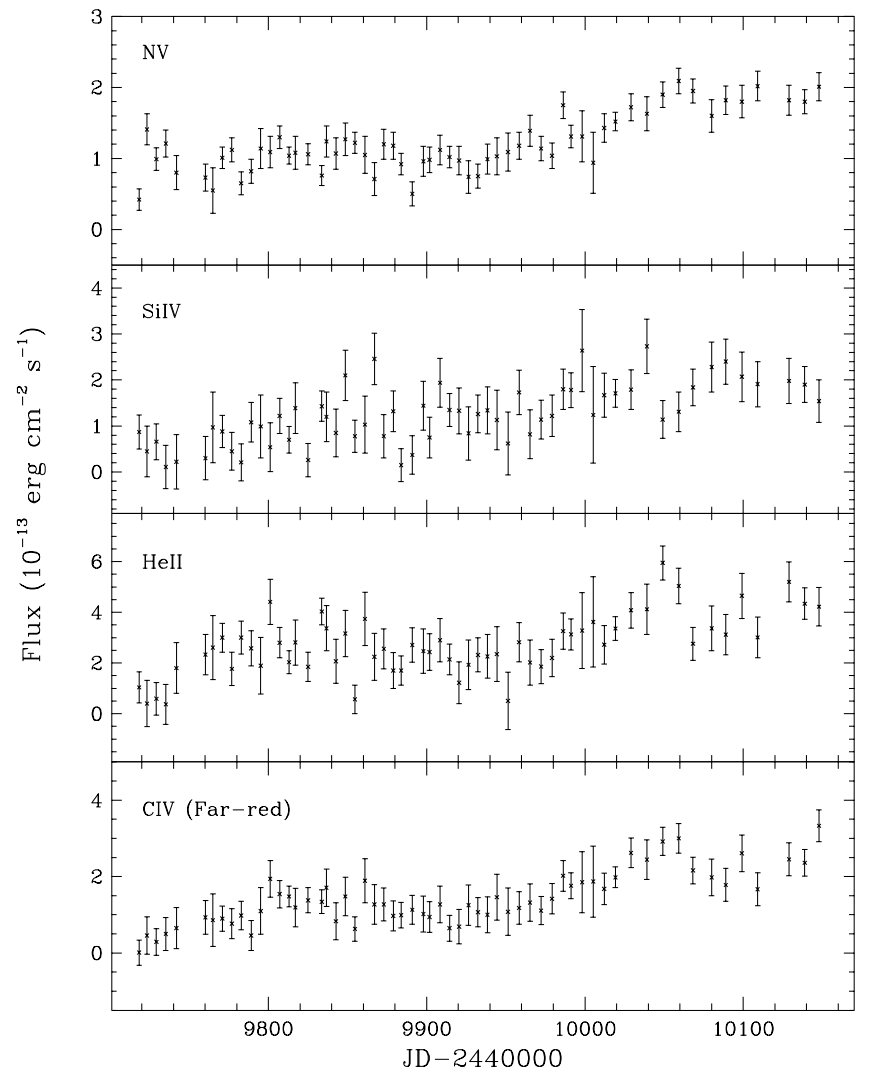

FIG. 4.- - Light curves for the weaker UV lines and C IV (far red)

uncertainties for $\operatorname{Ly} \alpha$ and $C$ IV slightly larger than those used here, but it gives uncertainties $20 \%-80 \%$ larger for the weaker lines. Either procedure should be considered as a conservative approach to calculating flux uncertainties, because any intrinsic variability between adjacent epochs will tend to increase the rms.

We used all the light curves to calculate the scale factors, with three exceptions: $F_{\lambda}(1370 \AA), F_{\lambda}(1855 \AA)$, and C IV (far red). The relatively large mean sampling interval for 3C 390.3 makes it likely that any rapidly varying spectral component will have varied between observations. This is likely for the UV continuum, and the cross-correlation results suggest it is also likely for C IV (far red). Therefore, for these three spectral components we rescaled using only those epochs within the monitoring period from JD 2,449, 810 to $2,449,890$. During this period, both the UV and X-ray continua (Leighly et al. 1997) were relatively quiescent. Using all the light curves would increase the mean uncertainties for $F_{\lambda}(1370 \AA)$ and $F_{\lambda}(1855 \AA)$ by $50 \%$ and $100 \%$, respectively.

\subsection{Variability Analysis}

Various parameters characterizing the continuum and emission-line variability are given in Table 5 . The mean flux, $\bar{F}$, and standard deviation, $\sigma_{F}$, have their usual meanings. The $R_{\max }$ parameter is the ratio of the maximum to minimum flux and is only useful when $\sigma_{F}$ is large compared to the measurement uncertainties. The values of $R_{\max }$ are particularly uncertain for Si IV $\lambda 1400$ and He II $\lambda 1640$. For C IV (far red), the very high $R_{\max }$ is due to the very low flux at the first epoch. If that point is ignored, $R_{\max }=7.24$.

The fourth parameter, $F_{\text {var }}$, in Table 5 is a measurementuncertainty-corrected estimate of the amplitude of the
TABLE 4

EMISSION-LINe FluXeS FOR N v $\lambda 1240$, Si IV $\lambda 1400$, He II $\lambda 1640$, AND C IV (FAR RED)

\begin{tabular}{|c|c|c|c|c|}
\hline \multirow{2}{*}{$\begin{array}{c}\text { JD } \\
(2,440,000+)\end{array}$} & \multicolumn{4}{|c|}{$\begin{array}{c}\text { FLUX } \\
\left(10^{-13} \mathrm{ergs} \mathrm{cm}^{-2} \mathrm{~s}^{-1}\right)\end{array}$} \\
\hline & $\mathrm{N} v \lambda 1240$ & Si IV $\lambda 1400$ & He II $\lambda 1640$ & $\mathrm{C}$ IV (far red) \\
\hline 9718.364 . & $0.42 \pm 0.15$ & $0.87 \pm 0.37$ & $1.04 \pm 0.61$ & $0.01 \pm 0.33$ \\
\hline 9723.306 . & $1.41 \pm 0.22$ & $0.45 \pm 0.55$ & $0.40 \pm 0.91$ & $0.46 \pm 0.49$ \\
\hline 9729.178 . & $0.99 \pm 0.16$ & $0.66 \pm 0.39$ & $0.59 \pm 0.64$ & $0.29 \pm 0.35$ \\
\hline 9735.186 & $1.21 \pm 0.19$ & $0.11 \pm 0.47$ & $0.37 \pm 0.79$ & $0.50 \pm 0.43$ \\
\hline $9741.873 \ldots$ & $0.80 \pm 0.24$ & $0.22 \pm 0.59$ & $1.80 \pm 1.00$ & $0.65 \pm 0.54$ \\
\hline 9760.255 . & $0.73 \pm 0.19$ & $0.30 \pm 0.47$ & $2.33 \pm 0.79$ & $93 \pm 0.44$ \\
\hline 9764.905. & $0.55 \pm 0.32$ & $0.97 \pm 0.77$ & $2.61 \pm 1.26$ & $0.86 \pm 0.69$ \\
\hline $9770.886 \ldots$ & $1.01 \pm 0.15$ & $0.88 \pm 0.35$ & $3.00 \pm 0.57$ & $0.90 \pm 0.33$ \\
\hline 9776.887 . & $1.12 \pm 0.17$ & $0.45 \pm 0.41$ & $1.77 \pm 0.66$ & $0.77 \pm 0.39$ \\
\hline 9782.807. & $0.65 \pm 0.16$ & $0.21 \pm 0.40$ & $3.00 \pm 0.65$ & $0.98 \pm 0.37$ \\
\hline 9789.148 . & $0.82 \pm 0.17$ & $1.08 \pm 0.43$ & $2.58 \pm 0.70$ & $6 \pm 0.39$ \\
\hline 9795.146. & $1.14 \pm 0.28$ & $0.99 \pm 0.68$ & $1.89 \pm 1.12$ & \\
\hline 9801.148. & $1.09 \pm 0.22$ & $0.54 \pm 0.53$ & $4.41 \pm 0.89$ & \\
\hline $9807.135 \ldots$ & $1.30 \pm 0.16$ & $1.22 \pm 0.38$ & $2.80 \pm 0.60$ & $1.54 \pm 0.36$ \\
\hline 9813.066. & $1.04 \pm$ & $0.70 \pm 0.29$ & $2.03 \pm 0.45$ & \\
\hline 9817.068 & $1.08 \pm 0$ & $1.39 \pm 0.55$ & 89 & \\
\hline 9825.052 & $6 \pm 0.15$ & $0.26 \pm 0.36$ & 1. & \\
\hline 9833.729 . & $0.76 \pm 0.14$ & $1.43 \pm 0.33$ & $4.03 \pm 0.53$ & \\
\hline $9836.729 \ldots$ & $1.24 \pm 0.22$ & $1.20 \pm 0.54$ & $3.37 \pm 0.89$ & \\
\hline 9842.651. & $1.07 \pm$ & $0.85 \pm$ & 87 & \\
\hline 9848.643 . & $1.27 \pm 0.23$ & $2.10 \pm 0.55$ & 91 & \\
\hline 9854.650 . & $1.22 \pm 0.15$ & $0.78 \pm 0.35$ & $0.57 \pm 0.56$ & $0.63 \pm 0.32$ \\
\hline 9861.013. & $1.05 \pm 0.26$ & $1.03 \pm 0.62$ & $3.74 \pm 1.05$ & $1.89 \pm 0.58$ \\
\hline 9866.988 . & & & 2. & \\
\hline 9872.888 & $1.20=$ & $0.78=$ & \pm 0 & \\
\hline 9878.882 & $1.18 \pm$ & $1.32 \pm 0.44$ & 1.7 & \\
\hline $9883.897 \ldots$ & $0.92 \pm 0.15$ & $0.15 \pm 0.36$ & $1.70 \pm 0.58$ & $0.99 \pm 0.33$ \\
\hline $9890.896 \ldots$ & & $0.37 \pm 0.42$ & & \\
\hline 9897.8 & & 1.44 & 2. & \\
\hline 9901.807 . & $0.98 \pm$ & $0.75 \pm 0.44$ & \pm 0.72 & 0.94 \\
\hline 9908.449 . & 21 & $1.94 \pm 0.53$ & $2.90 \pm 0$ & $1.27 \pm 0.48$ \\
\hline 9914.440 .. & $1.02 \pm 0.15$ & $1.35 \pm 0.36$ & $2.14 \pm 0.60$ & $0.65 \pm 0.34$ \\
\hline $9920.455 .$. & & & 1. & \\
\hline 9926.450 . & 0.74 & 0.84 & 1.9 & \\
\hline 9932.421. & $0.75 \pm$ & $1.26 \pm 0.41$ & $2.32 \pm 0$ & \\
\hline $9938.406 .$. & $0.99 \pm 0.21$ & $1.34 \pm 0.51$ & $2.26 \pm 0.86$ & $1.00 \pm 0.47$ \\
\hline $9944.407 .$. & & & & \\
\hline 9951.402 . & 1.09 & 0.62 & 0. & \\
\hline $9958.412 \ldots$ & $1.18=$ & $1.73\rfloor$ & $2.82 \pm 0.77$ & \\
\hline 9965.314 . & $39 \pm 0.22$ & $0.82 \pm 0.53$ & $2.02 \pm 0.89$ & \pm 0.49 \\
\hline $9972.313 \ldots$ & $1.14 \pm 0.17$ & $1.14 \pm 0.42$ & $1.86 \pm 0.67$ & $1.11 \pm 0.37$ \\
\hline $9979.223 \ldots$ & & & 2.2 & \\
\hline $9986.234 \ldots$ & & 1.80 & \pm 0.72 & \\
\hline $9991.216 .$. & $1.31 \pm 0.16$ & $1.78 \pm 0.38$ & $3.13 \pm 0.61$ & \\
\hline $9998.246 \ldots$ & $1.31 \pm 0.36$ & $2.64 \pm 0.89$ & $3.28 \pm 1.49$ & $1.85 \pm 0.80$ \\
\hline 10005.241. & $0.94 \pm 0.43$ & $1.24 \pm 1.05$ & $3.62 \pm 1.78$ & \\
\hline & & & $2.72 \pm 0$ & \\
\hline 1001 & $1.52 \pm$ & $1.71 \pm 0$ & $3.36 \pm 0.47$ & \\
\hline $10029.144 .$. & $1.72 \pm 0.19$ & $1.79 \pm 0.43$ & $4.08 \pm 0.70$ & $2.62 \pm 0.39$ \\
\hline $10039.148 \ldots$ & $1.63 \pm 0.24$ & $2.73 \pm 0.59$ & $4.12 \pm 0.99$ & $2.44 \pm 0.52$ \\
\hline & & $1.14 \pm 0.41$ & $5.95 \pm 0.67$ & \\
\hline & $2.09 \pm 0$ & $1.31 \pm 0.43$ & $5.04 \pm 0$ & \\
\hline $10068.253 .$. & & $1.84 \pm 0.40$ & $2.76 \pm 0$ & \\
\hline $10080.050 \ldots$ & $1.60 \pm 0.23$ & $2.28 \pm 0.54$ & $3.37 \pm 0.88$ & $1.98 \pm 0.48$ \\
\hline $10088.975 \ldots \ldots$ & $1.82 \pm 0.20$ & $2.40 \pm 0.49$ & $3.12 \pm 0.80$ & $1.78 \pm 0.43$ \\
\hline 10090165 & $1.80 \pm 0.23$ & $2.07 \pm 0.54$ & $4.65 \pm 0.89$ & \\
\hline & $2.02 \pm 0.21$ & & & \\
\hline $10128.916 \ldots$ & & $1.98 \pm 0.49$ & $5.20 \pm 0.79$ & $2.45 \pm 0.43$ \\
\hline $10138.876 \ldots \ldots$ & $1.80 \pm 0.17$ & $1.90 \pm 0.39$ & $4.34 \pm 0.62$ & $2.36 \pm 0.35$ \\
\hline $10147.854 \ldots$ & $2.01 \pm 0.20$ & $1.54 \pm 0.46$ & $4.22 \pm 0.76$ & $3.33 \pm 0.42$ \\
\hline
\end{tabular}

variability relative to the mean flux, defined as

$$
F_{\mathrm{var}}=\frac{\sqrt{\left(\sigma_{F}^{2}-\Delta^{2}\right)}}{\bar{F}},
$$

where $\Delta^{2}$ is the mean square value of the individual mea- 
TABLE 5

\begin{tabular}{|c|c|c|c|c|}
\hline Feature & $\bar{F}^{\mathrm{a}}$ & ${\sigma_{F}}^{\mathrm{a}}$ & $R_{\max }$ & $F_{\mathrm{var}}$ \\
\hline$F_{\lambda}(1370 \AA ̊) \ldots \ldots$ & 6.33 & 2.99 & 7.33 & 0.46 \\
\hline$F_{\lambda}(1855 \AA) \ldots \ldots \ldots$ & 4.57 & 1.95 & 7.62 & 0.41 \\
\hline $\operatorname{Ly} \alpha \ldots \ldots \ldots$ & 12.22 & 2.10 & 2.11 & 0.14 \\
\hline $\operatorname{Ly} \alpha$ (blue) .... & 3.55 & 0.74 & 2.61 & 0.15 \\
\hline Ly $\alpha$ (center)... & 6.72 & 0.93 & 2.08 & 0.10 \\
\hline Ly $\alpha$ (red) .............. & 2.79 & 0.75 & 2.71 & 0.24 \\
\hline $\mathrm{C}$ IV $\lambda 1549 \ldots$ & 9.56 & 2.92 & 3.97 & 0.29 \\
\hline C Iv (blue) ... & 3.81 & 1.24 & 4.63 & 0.31 \\
\hline $\mathrm{C}$ IV (center) ....... & 4.06 & 1.07 & 3.24 & 0.25 \\
\hline $\mathrm{C}$ iv (red).......... & 2.11 & 0.86 & 6.44 & 0.39 \\
\hline $\mathrm{N} v i 1240 \ldots \ldots \ldots$ & 1.21 & 0.41 & 4.97 & 0.29 \\
\hline Si IV $\lambda 1400 \ldots \ldots \ldots$ & 1.24 & 0.65 & $(24.82)$ & 0.33 \\
\hline $\mathrm{C}$ IV (far-red)...... & 1.42 & 0.70 & $(330.00)$ & 0.37 \\
\hline He II $\lambda 1640 \ldots \ldots$. & 2.70 & 1.19 & (16.08) & 0.31 \\
\hline
\end{tabular}

${ }^{a}$ Units are $10^{-15} \mathrm{ergs} \mathrm{cm}^{-2} \mathrm{~s}^{-1} \AA^{-1}$ for continuum fluxes and $10^{-13} \mathrm{ergs} \mathrm{cm}^{-2} \mathrm{~s}^{-1}$ for emission lines.

surement uncertainties, $\epsilon_{i}$, for the $i=1,2,3, \ldots, N$ observations; i.e.,

$$
\Delta^{2}=\frac{1}{N} \sum_{i=1}^{N} \epsilon_{i}^{2} .
$$

Both UV continuum light curves show several distinct features that are also seen, although more clearly defined, in the ROSAT X-ray light curve of 3C 390.3 (Leighly et al. 1997). The UV continuum rises at the beginning of the campaign, followed by a flare at JD 2,449,795 that coincides with a large X-ray flare. The X-ray flare lasted about 15 days, so if the UV flare were of comparable length we would expect only a single point above the general trend in the data, as observed. After the flare the continuum shows marginal evidence for flickering (the "quiescent period"), followed by a dip that is more pronounced at shorter wavelengths. The UV continuum then rises to a maximum around JD 2,450,020, followed by a small decline and then another rise at the end of the monitoring campaign.

The amplitude of the continuum variability is only slightly lower in the longer wavelength band. In Figure 5 we plot $F_{\lambda}(1855 \AA)$ versus $F_{\lambda}(1370 \AA)$ along with a straight-line fit that allows for errors in both variables. As found for Fairall 9 (Rodríguez-Pascual et al. 1997), the straight-line fit is very good $\left(\chi_{v}^{2}=1.4\right)$, indicating that there is no significant change in UV continuum shape with luminosity. The small positive $y$-intercept may indicate a contribution to $F_{\lambda}(1855 \AA)$ from weak, blended $\mathrm{Fe}$ II emission lines and Balmer continua of $(0.5 \pm 0.2) \times 10^{-15} \mathrm{ergs} \mathrm{cm}^{-2} \mathrm{~s}^{-1} \AA^{-1}$. This small value $\left[10 \%\right.$ of the mean $\left.F_{\lambda}(1855 \AA)\right]$ and the absence of detectable $\mathrm{Fe}$ II in the rms spectrum (Fig. 1) show that the $F_{\lambda}(1855 \AA)$ variability parameters have not been significantly affected by a constant of weakly varying emission-line contamination. Fitting to the logarithm of the continuum fluxes gives

$$
\begin{aligned}
\log F_{\lambda}(1855 \AA)= & (-0.15 \pm 0.20)+(1.02 \pm 0.26) \\
& \times \log F_{\lambda}(1370 \AA),
\end{aligned}
$$

consistent with a constant UV continuum shape $\left(f_{v} \propto v^{-1}\right)$ during the campaign.

All of the emission-line light curves show a similar trend to that of the continuum, with an overall increase in flux

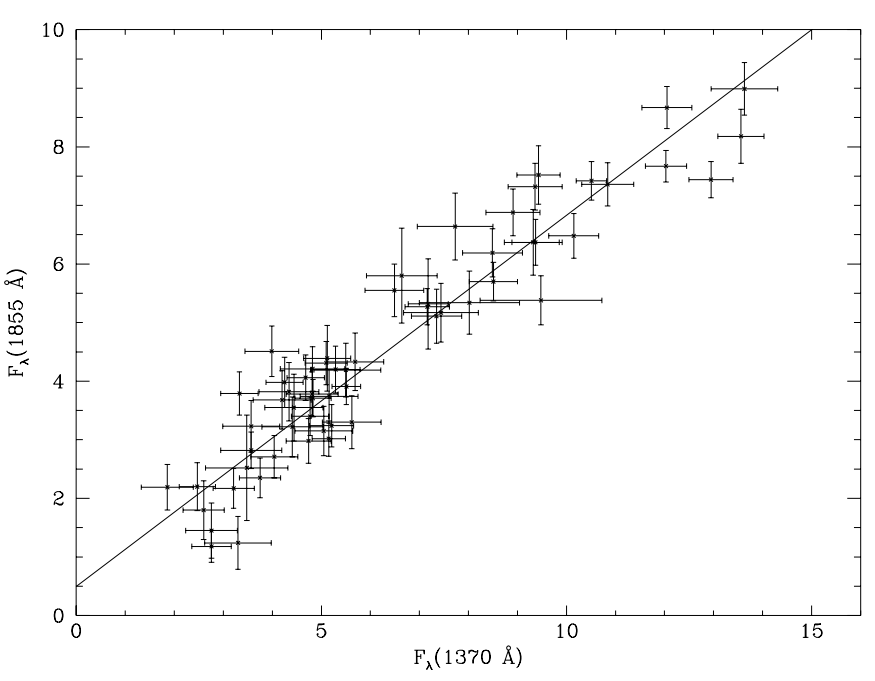

FIG. 5.-Correlation between $F_{\lambda}(1370 \AA)$ and $F_{\lambda}(1855 \AA)$ in units of $10^{-15} \mathrm{ergs} \mathrm{cm}^{-2} \mathrm{~s}^{-1} \AA^{-1}$. The solid line is a straight-line fit allowing for errors on both variables.

through the campaign. The smallest $F_{\text {var }}$ among the emission lines occurs for $L y \alpha$, while the other lines vary with similar amplitudes. Correcting for the narrow-line components ( $\$ 3.1$ ) would increase $F_{\text {var }}$ by $\sim 0.04$ for the Ly $\alpha$ and C IV line centers, making them comparable to those derived for the blue wings but lower than for the red wings. Unlike the $\mathrm{C}$ IV line wings, $F_{\text {var }}$ for $\mathrm{Ly} \alpha$ (blue) is almost half that for $\operatorname{Ly} \alpha$ (red).

Wamsteker et al. (1997) found that the C IV/Ly $\alpha$ ratio for both the blue and red wings increased with increasing continuum and $\mathrm{C}$ IV flux in the $I U E$ archival data but appeared to "saturate" for $F(\mathrm{C}$ IV red $)$ or $F(\mathrm{C}$ IV blue $)>5 \times 10^{-13}$ ergs $\mathrm{cm}^{-2} \mathrm{~s}^{-1}$. In Figure 6 we plot the $\mathrm{C}$ IV/Ly $\alpha$ ratio correlations for both line wings using the data from Table 3 . Correlating against the line flux minimizes the effect of time delays in the line response, which otherwise increase the scatter. All four correlations shown in Figure 6 are significant, with Spearman rank correlation coefficients $>0.993$.

As for the variability amplitudes, there is some evidence for contrasting behavior in the $\mathrm{C}$ IV $/ \mathrm{Ly} \alpha$ ratio between the red and blue wings. For $\mathrm{C}$ IV line-wing fluxes $>3 \times 10^{-13}$ ergs $\mathrm{cm}^{-2} \mathrm{~s}^{-1}$, the $\mathrm{C} \mathrm{IV} / \mathrm{Ly} \alpha$ ratio for the red wing falls systematically at the lower end of the range for the blue wing (Fig. 6, top panels), whereas the data for both wings overlap at lower flux levels. Although this gives some support to the idea of saturation at high flux levels in the red wing, the trend is not as apparent as for the archival data. A difference between the blue and red wings is more apparent from the correlation with continuum flux, for which the $\mathrm{C}$ IV/Ly $\alpha$ ratio is systematically higher in the blue wing than the red (Fig. 6, bottom panels). This trend is not seen in the archival data. Overall, compared to either the continuum flux or the line-wing flux, the line fluxes are lower and the average $\mathrm{C}$ IV/Ly $\alpha$ ratio is higher than found for the archival data. This is particularly true for the blue wing, for which the mean ratio is $50 \%$ higher. These results suggest a change in the behavior of the line-emitting gas. We will return to this point in the discussion.

\subsection{Cross-Correlation Analysis}

The time delay or lag of the emission-line variation relative to variations in the ionizing continuum is usually quantified by use of a cross-correlation analysis. We performed 

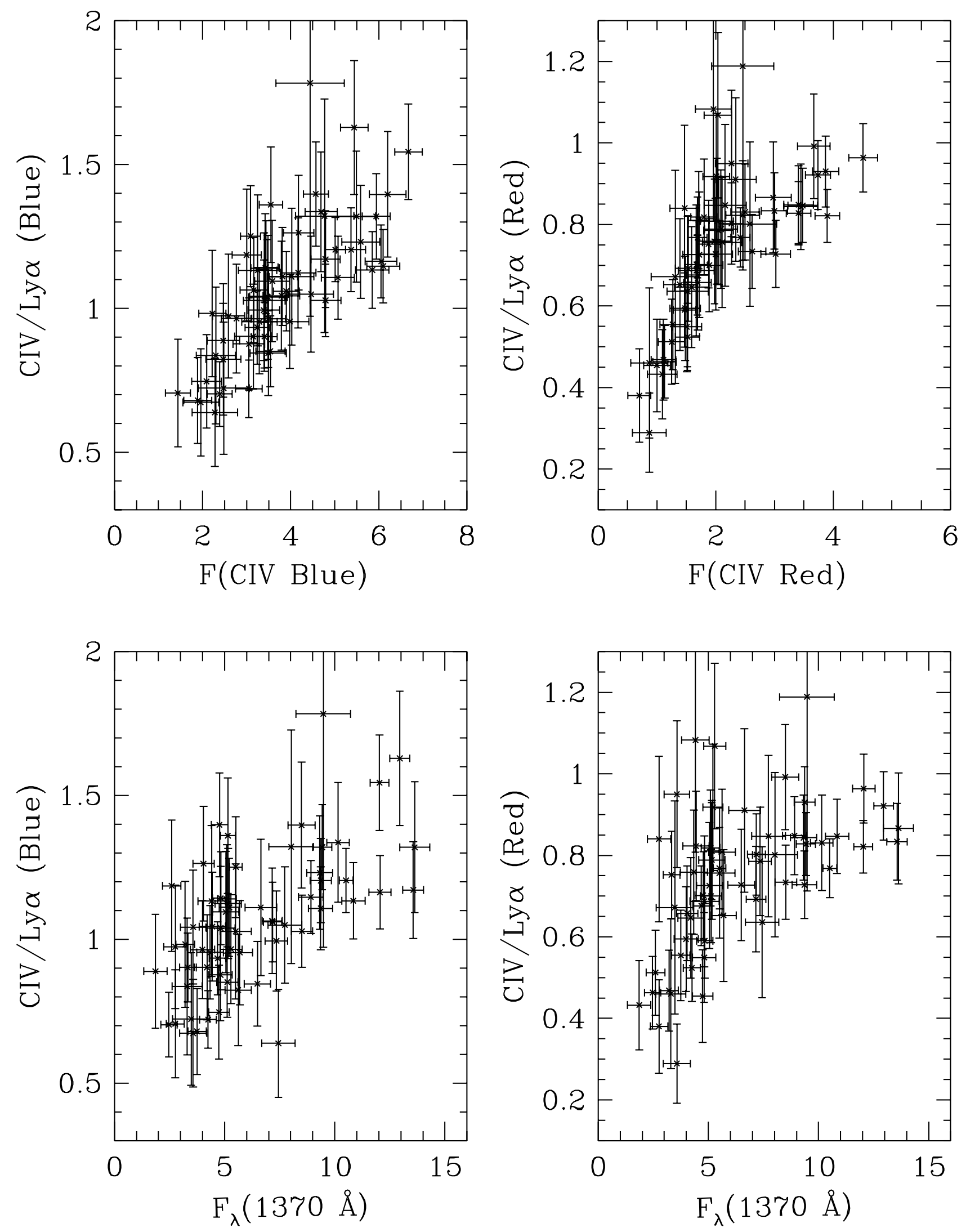

FIG. 6. - Correlation between the $\mathrm{C}$ IV/Ly $\alpha$ ratio for the blue and red wings as a function of line-wing flux and $F_{\lambda}(1370 \AA)$

two such analyses, using the interpolation cross-correlation function (ICCF) as implemented by White \& Peterson (1994) and their implementation of the discrete crosscorrelation function (DCF) of Edelson \& Krolik (1988). The cross-correlation function (CCF) results are tabulated in Table 6 and shown in Figure 7. The ICCF and DCF results are identical to within the DCF error bars. Results that are particularly uncertain are given within brackets in Table 6 . 
TABLE 6

\begin{tabular}{|c|c|c|c|c|c|c|}
\hline \multicolumn{7}{|c|}{ Cross-CORRELATION Results } \\
\hline Feature & $r_{\max }$ & $\begin{array}{c}\text { FWHM } \\
\text { (days) }\end{array}$ & $\begin{array}{c}\tau_{\text {peak }} \\
\text { (days) }\end{array}$ & $\begin{array}{c}\tau_{\text {cent }} \\
\text { (days) }\end{array}$ & $\begin{array}{c}\tau_{\mathrm{FR}} \pm \sigma_{\mathrm{FR}} \\
\text { (days) }\end{array}$ & $\begin{array}{c}\tau_{\text {FR/RSS }} \pm \sigma_{\text {FR/RSS }} \\
\text { (days) }\end{array}$ \\
\hline$F_{\lambda}(1370 \AA)(\mathrm{ACF}) \ldots \ldots$ & 1.00 & 47 & 0 & 0 & $\ldots$ & $\ldots$ \\
\hline$F_{\lambda}(1855 \AA)(\mathrm{ACF}) \ldots . .$. & 1.00 & 58 & 0 & 0 & $\ldots$ & $\ldots$ \\
\hline$F_{\lambda}(1855 \AA) \ldots \ldots \ldots \ldots \ldots$ & 0.95 & 61 & -2 & 3 & $-10 \pm 11$ & $-3 \pm 18$ \\
\hline $\operatorname{Ly} \alpha \ldots \ldots \ldots \ldots \ldots \ldots$ & 0.80 & 278 & 93 & 67 & $59 \pm 12$ & $57 \pm 22$ \\
\hline Ly $\alpha$ (blue) .............. & 0.73 & $\ldots$ & 56 & 62 & $(61 \pm 25)$ & $(66 \pm 35)$ \\
\hline Ly $\alpha$ (center) ............. & 0.78 & 214 & 83 & 65 & $63 \pm 18$ & $63 \pm 26$ \\
\hline $\operatorname{Ly} \alpha($ red $) \ldots \ldots \ldots \ldots \ldots$ & 0.85 & $\cdots$ & 26 & 54 & $54 \pm 9$ & $51 \pm 17$ \\
\hline $\mathrm{C}_{\text {IV }} \lambda 1549 \ldots \ldots \ldots \ldots$ & 0.88 & $\ldots$ & 15 & 41 & $40 \pm 7$ & $36 \pm 14$ \\
\hline $\mathrm{C}$ iv (blue) ............. & 0.88 & $\ldots$ & 45 & 41 & $34 \pm 9$ & $35 \pm 14$ \\
\hline $\mathrm{C}$ IV (center)...$\ldots \ldots \ldots$ & 0.85 & $\ldots$ & 3 & 50 & $45 \pm 8$ & $40 \pm 17$ \\
\hline $\mathrm{C}$ IV (red) $\ldots \ldots \ldots \ldots \ldots$ & 0.89 & $\cdots$ & 16 & 35 & $36 \pm 9$ & $34 \pm 16$ \\
\hline N v $\lambda 1240 \ldots \ldots \ldots \ldots \ldots$ & 0.87 & $\ldots$ & 15 & 43 & $36 \pm 13$ & $34 \pm 17$ \\
\hline Si IV $\lambda 1400 \ldots \ldots \ldots \ldots$ & $(0.70)$ & $\ldots$ & $(-27)$ & (9) & $(5 \pm 27)$ & $(7 \pm 31)$ \\
\hline $\mathrm{C}$ IV (far-red) ............ & 0.89 & 202 & 9 & 11 & $11 \pm 9$ & $11 \pm 14$ \\
\hline Нe II $\lambda 1640 \ldots \ldots \ldots \ldots$ & $(0.73)$ & $(177)$ & (4) & $(12)$ & $(9 \pm 11)$ & $(14 \pm 18)$ \\
\hline
\end{tabular}

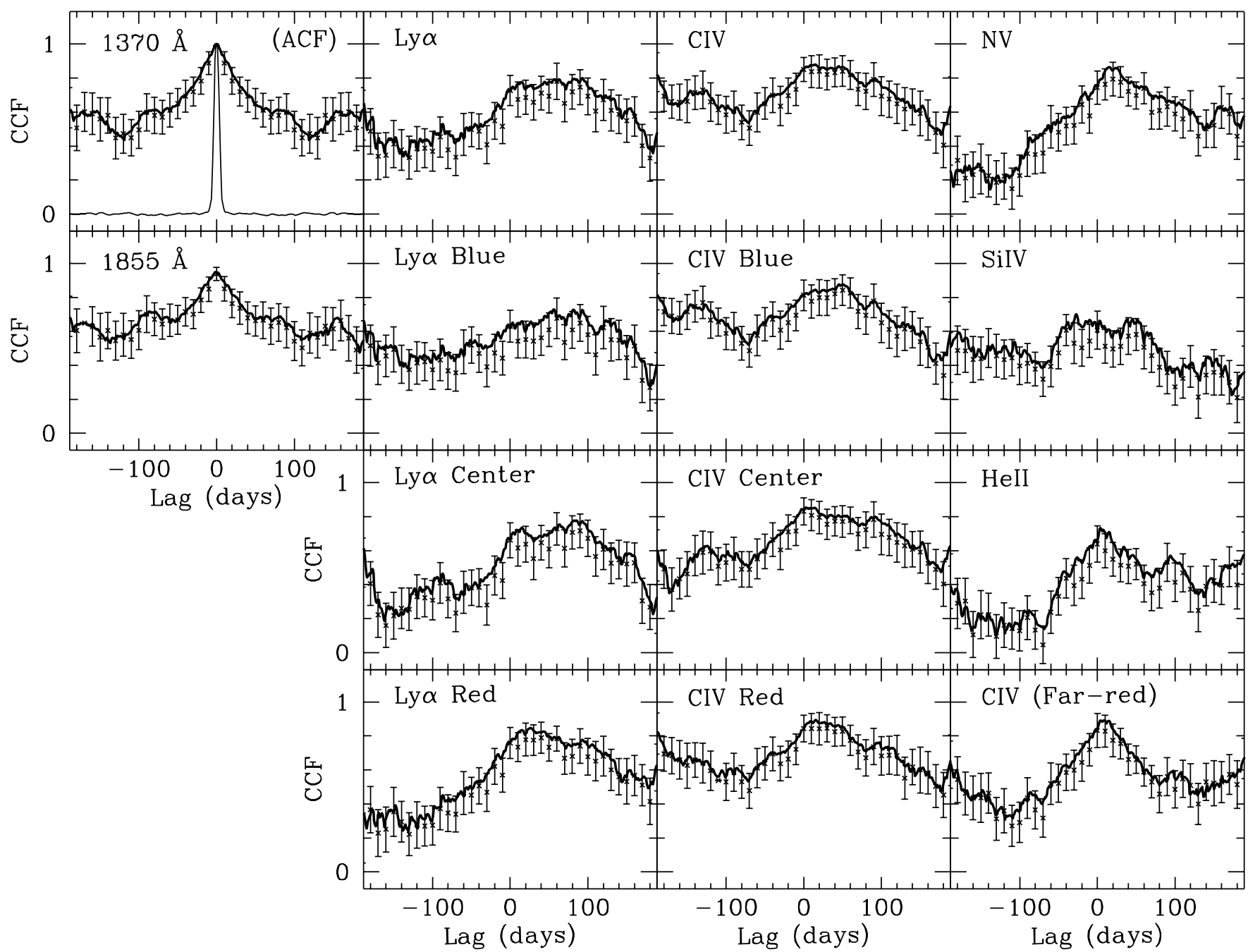

FIG. 7.-Continuum and emission-line ICCFs (solid lines) and DCFs (error bars). The top left box shows the $F_{\lambda}(1370 \AA$ ) ACF and the sampling window ACF. The other light curves were all cross-correlated with that of $F_{\lambda}(1370 \AA)$. 
Because of the "flat-topped" nature of many of the CCFs for 3C 390.3 and the relatively large flux uncertainties, the position of the CCF peak, $\tau_{\text {peak }}$, is highly uncertain. Therefore, for this AGN we consider that the CCF centroid, $\tau_{\text {cent }}$, is a more stable and representative measure of the emissionline lags. The centroids quoted in Table 6 were calculated at a level of 0.7 times the maximum CCF correlation coefficient, $r_{\max }$. Adopting a level of $0.8 r_{\max }$, as used in previous papers (e.g., Wanders et al. 1997), usually gave a much larger uncertainty on $\tau_{\text {cent }}$ because of the uncertainty on the value and location of $r_{\max }$.

There is no clear consensus on how best to assign uncertainties to CCF parameters. Previous procedures have included comparison of different CCF methods and analytical estimates (see Gaskell 1994 and references therein). Here we have adopted a Monte Carlo approach, in which large numbers of surrogate data generated from the original data were cross-correlated using the ICCF. The distribution of centroids calculated from these surrogate data ICCFs - the cross-correlation probability distribution (CCPD; Maoz \& Netzer 1989) - then gives an estimate of the uncertainties (Peterson et al. 1998). The surrogate data were generated in two ways:

1. Randomly generated, normally distributed errors (Gaussian deviates) derived from the observed flux uncertainties were added to the observed fluxes. These data were then cross-correlated. This is the "flux randomization" (FR) method described in Peterson et al. (1998).

2. Gaussian deviates were added and data points were randomly excluded before cross-correlation. This is the FR/RSS method, where the second step involves " random subset selection" (RSS) (Peterson et al. 1998), which gives an estimate of the effect of "errant" data that may unduly influence the CCF.

Peterson et al. (1997) find that CCPDs calculated using the FR method alone tend to underestimate the true uncertainty, whereas the FR/RSS method is conservative in that it excludes real data.

We constructed CCPDs for 1000 runs of both the FR and FR/RSS methods and from these calculated the mean centroids and standard deviations. These are given in Table 6 as $\tau_{\mathrm{FR}} \pm \sigma_{\mathrm{FR}}$ and $\tau_{\mathrm{FR} / \mathrm{RSS}} \pm \sigma_{\mathrm{FR} / \mathrm{RSS}}$. For some of the weaker emission lines and for $\operatorname{Ly} \alpha$ (blue), it was necessary to exclude some outliers ( $\approx 10 \%$ of the runs) that lie in "alias peaks," spaced at approximately $\tau=0 \pm 160$ days, caused by features spaced at those intervals in the second half of the continuum light curves. These alias peaks are not seen in either the $\mathrm{C}$ IV or the other Ly $\alpha$ CCPDs. The data for lines where aliasing was a particular problem, leading to greater uncertainty in their true centroids, are bracketed in Table 6. We note that using a standard deviation to describe the uncertainty is only appropriate if the CCPD is Gaussian in shape, which is the case here. Gaskell (1988b) and Maoz \& Netzer (1989) suggest taking the range of lags within which $68 \%$ of the centroids contributing to the CCPDs occur. The median difference between half the ranges calculated using that procedure and the $\sigma$ values given in Table 6 is only 1.5 days.

\subsubsection{The UV Continuum}

The sampling window autocorrelation function (ACF) shown in the top left window in Figure 7 is clearly narrower than the continuum ACF. This illustrates that the chosen sampling intervals were adequate to follow most of the power in the continuum light curves. The mean sampling interval of 7.2 days limits the detection of lags on small timescales between $F_{\lambda}(1370 \AA)$ and $F_{\lambda}(1855 \AA)$, but the cross-correlation results are consistent with no lag.

\subsubsection{The UV Emission Lines}

The Ly $\alpha$ and $\mathrm{C}$ IV emission-line light curves do not show the shortest timescale features seen in the continuum light curves. This suggests a fairly large lag, as confirmed by the cross-correlation analysis. The CCF amplitude remains high at large lags because of the linear trends in the light curves. Removing these trends reduces the CCF amplitudes but does not significantly alter the values of $\tau_{\text {cent }}$.

The lags are larger for $\mathrm{Ly} \alpha$ than for C IV. We find no evidence for a significant lag between the blue and red wings of $\mathrm{C} \mathrm{IV}$, and the line center also shows no significant lag relative to the wings. The Ly $\alpha$ data are consistent with these conclusions, although more uncertain. The C IV (far-red) component can be used to further search for velocitydependent lags. The velocity limits for this component were chosen to cover the same velocity interval $(\approx 6000-11,000$ $\mathrm{km} \mathrm{s}^{-1}$ ) relative to the $\mathrm{C}$ IV line center as does the $\mathrm{N} \mathrm{v}$ $\lambda 1240$ measurement relative to $\operatorname{Ly} \alpha(\S 3.1)$. This velocity region overlaps with $\mathrm{He}$ II $\lambda 1640$, but the shape of the rms spectrum strongly suggests the feature is not associated with He II $\lambda 1640$. A cross-correlation analysis on the resultant C IV (far-red) light curve (Fig. 4) shows a smaller lag than for the rest of C IV. Repeating this procedure in the blue wing of $\mathrm{C}$ IV produces no significant difference in lag. Unfortunately, the possible contaminating effect of $\mathrm{N} \mathrm{V}$ $\lambda 1240$ confuses the issue for Ly $\alpha$.

\section{DISCUSSION}

The UV data for 3C 390.3 clearly show that both the continuum and broad emission lines are highly variable. The detailed variability characteristics show some similarities and some contrasts to those of the intensively monitored Seyfert 1 galaxies.

The amplitude of the continuum variability is slightly lower in the longer wavelength UV wave band according to the $F_{\text {var }}$ parameter, but the difference is smaller than that seen in some Seyfert 1 galaxies (e.g., Clavel et al. 1991; Crenshaw et al. 1996; Wanders et al. 1997). Over the same nine month period in $1995, F_{\text {var }} \approx 0.3$ for both the UV and X-ray continua (Leighly et al. 1997), suggesting that the similarity in variability amplitude may apply to the entire ionizing continuum. Such a similarity was not seen in NGC 4151 , for which the X-ray flux at $\sim 1.5 \mathrm{keV}$ varied by a factor of 3 more than the $1330 \AA$ flux (Edelson et al. 1996). In contrast, the optical continuum in 3C 390.3 appears to vary with a smaller amplitude than the UV (Dietrich et al. 1998), behavior consistent with that seen in some Seyfert 1 galaxies. In all of these AGN, this trend may be partly due to dilution of the nonstellar continuum by starlight from the host galaxy.

The broad UV emission-line variability correlates well with that of the UV continuum in 3C 390.3, but the emission-line lags and the $\mathrm{C}$ IV variability amplitude are larger than seen in Seyfert 1 galaxies of comparable UV, but lower X-ray, luminosity. For example, in NGC 5548 the Ly $\alpha$ lag is $\sim 10$ days (Clavel et al. 1991; Korista et al. 1995). The results for 3C 390.3 are consistent with the trend seen in Seyfert 1 galaxies for the higher ionization UV lines to vary 
on faster timescales. However, the distinction in behavior between what have been commonly referred to as "low" and "high" ionization lines $(\S 1)$ appears more complex in 3C 390.3. The derived lags for the Balmer lines $(\sim 20 \pm 8$ days; Dietrich et al. 1998) are smaller than those for both Ly $\alpha$ and C IV, a trend not seen in Seyfert 1 galaxies. Whether these contrasting characteristics are due to differences in the physical conditions in the BLR or are a consequence of differences in the shape and variability of the ionizing continuum is beyond the scope of this paper.

The difference in lag between 3C 390.3 and Seyfert 1 galaxies of comparable luminosity was noted by Wamsteker et al. (1997) based on IUE archival data, although the order-of-magnitude smaller mean sampling interval used here makes the result more secure. Wamsteker et al. found larger lags than those derived here, although even those may be underestimates. Applying the same analysis techniques used here to the measurements given by Wamsteker et al., we derive lags of $143 \pm 55$ and $116 \pm 60$ days for $L y \alpha$ and $\mathrm{C}$ IV, respectively, compared to the lags of $60 \pm 24$ and $37 \pm 14$ derived for the new data. Possible variations in lag have been found previously for Ly $\alpha$ in the Seyfert 1 galaxy Fairall 9 (Rodríguez-Pascual 1997) and for $\mathrm{H} \beta$ in NGC 5548 (Peterson et al. 1994). As for these AGN, the suggested variation in lag in $3 \mathrm{C} 390.3$ could be due to a change in the central ionizing luminosity (e.g., O'Brien, Goad, \& Gondhalekar 1995), although this seems unlikely since the mean UV continuum flux is only $30 \%$ lower for the new campaign. Other possibilities are that the physical properties of the emitting gas have changed, for which there is direct evidence (see below), or that the sampling rate of the archival data is insufficient to provide reliable lags.

The values of $F_{\text {var }}$ found here for the continuum, all of the $\mathrm{C}$ IV components, and $\mathrm{Ly} \alpha$ (red) are in good agreement with those derived by Wamsteker et al. (1997). In contrast, $F_{\text {var }}$ for $\operatorname{Ly} \alpha$ (blue) and $\operatorname{Ly} \alpha$ (center) are factors of 2 and 1.5 smaller, respectively, than for the archival data. As discussed in $\S 3.2$, the change in the $\mathrm{C}$ IV $/ \mathrm{Ly} \alpha$ ratio between the new and archival data is striking, particularly in the blue wing. The lower mean $\operatorname{Ly} \alpha$ flux is partly due to the lower mean continuum flux relative to the archival data, but even allowing for that, the efficiency of production of $\operatorname{Ly} \alpha$ photons in the new campaign is lower relative to C IV. Overall, the new data indicate a velocity-dependent change in the line emission, with $L y \alpha$ affected more than C IV.

Velocity-dependent changes in the UV line profiles in 3C 390.3 were noted previously by Zheng (1996) and Wamsteker et al. (1997). Zheng found that, although the Ly $\alpha$ and C IV blue wings were usually stronger than the red in the IUE archival data, there were some very rare epochs when the opposite was true. During the new campaign the blue wing was always strongest. There is growing evidence for long-term variability in the BLR gas properties and/or distribution. This evidence mainly comes from long-term studies of Balmer-line variability in Seyfert 1 galaxies (Wanders 1997), but there is also some evidence from UV data (e.g., in NGC 5548; Goad \& Koratkar 1998).

We find no significant lag between the blue and red wings of the Ly $\alpha$ and C IV lines in either the new or archival data (adopting the larger uncertainties calculated for the archival data using the FR/RSS method). The new Balmer line data also show no evidence for radial motion (Dietrich et al.
1998). These results imply that radial motion does not dominate the kinematics of the UV/optical line-emitting gas in 3C 390.3. The lack of a lag between the blue and red wings is broadly consistent with the predictions of an accretion disk model. Detailed modeling is required to determine whether the different relative responses of the blue and red wings of the Ly $\alpha$ and C IV lines, at least at some epochs, the multiple-components in the Balmer lines (Dietrich et al.), and the C IV (far red) and possibly related Balmer line components can be explained by such a model.

\section{SUMMARY}

As part of an extensive multiwavelength monitoring campaign, the broad-line radio galaxy 3C 390.3 was observed every 6-10 days during the period 1994 December 31-1996 March 5. Large-amplitude continuum and broad emissionline variability was detected. The principal conclusions are as follows:

1. The UV continuum varied by a factor of 7 , with little difference in amplitude between 1370 and $1855 \AA$. During 1995, both the shape and amplitude of the UV continuum light curve are very similar to that of the X-ray light curve observed by ROSAT. This is in contrast to the previously monitored Seyfert 1 galaxies in which the $\mathrm{X}$-ray amplitude is usually significantly larger than the UV. During the same period, the optical continuum variability amplitude in 3C 390.3 was smaller than that of the UV, behavior similar to that seen in Seyfert 1 galaxies.

2. The broad emission lines show correlated variability with that of the UV continuum, with a general increase in flux through the campaign. The Ly $\alpha$ and $\mathrm{C}$ IV emission-line variations lag the continuum by 35-70 days. Overall, the emission-line lags are systematically larger than those derived for Seyfert 1 galaxies of comparable UV luminosity.

3. The trend in lag seen for the higher ionization UV lines is consistent with the idea of BLR ionization stratification found for Seyfert 1 galaxies, although the uncertainties are greater for the weaker lines in 3C 390.3. However, the Ly $\alpha$ and $C$ Iv lags are significantly larger than those found for the Balmer lines, in contrast to the results found for Seyfert 1 galaxies, blurring the usual distinction between "high" and "low" ionization lines.

4. No evidence is found for a lag between the red and blue wings of C IV or Ly $\alpha$, implying that radial motion does not dominate the kinematics of the high-ionization lineemitting BLR gas.

5. Comparing the new IUE data with archival data, there is some evidence that the BLR in 3C 390.3 evolves. The relative strength of the blue and red wings in $L y \alpha$ and C IV $\lambda 1549$ varies with time, although the double-peaked structure, seen in both the UV and optical line profiles, remains.

We thank the staff at the VILSPA and Goddard IUE observatories for their outstanding work on our behalf. We also gratefully acknowledge the support of many colleagues, including those on the IUE time-allocation committees. This work has been supported in part by grant SFB328D (Landessternwarte Heidelberg), NASA grants NAG 5-3233 and NAG 5-3497 to Ohio State University, and grant 97-02-17625 of the Russian Basic Research Foundation. 
REFERENCES

Alef, W., Wu, S. Y., Preuss, E., Kellermann, K. I., \& Qui, Y. H. 1996, A\&A, 308,376

Alloin, D., et al. 1995, A\&A, 293, 293

Barr, P., et al. 1980, MNRAS, 193, 562

Blandford, R. D., \& McKee, C. F. 1982, ApJ, 255, 419

Chen, K., Halpern, J. P., \& Filippenko, A. V. 1989, ApJ, 339, 742

Clavel, J. C., \& Wamsteker, W. 1987, ApJ, 320, L9

Clavel, J. C., et al. 1991, ApJ, 366, 64

Collier, S., et al. 1998, ApJ, 500, 162

Crenshaw, D. M., et al. 1996, ApJ, 470, 322

Dietrich, M., et al. 1993, ApJ, 408, 416 .1998, ApJS, 115, 185

Edelson, R. A., \& Krolik, J. H. 1988, ApJ, 333, 646

Edelson, R. A., et al. 1996, ApJ, 470, 364

Eracleous, M., Halpern, J. P., Gilbert, A. M., Newman, J. A., \& Filippenko, A. V. 1997, ApJ, 490, 216

Eracleous, M., Halpern, J. P., \& Livio, M. 1996, ApJ, 459, 89

Eracleous, M., Halpern, J. P., Livio, M., \& Filippenko, A. V. 1998, in preparation

Gaskell, C. M. 1988a, in Active Galactic Nuclei, ed. H. R. Miller \& P. J. Wiita (Berlin: Springer), 61 1988b, ApJ, 325, 114

. 1994, in ASP Conf. Ser. 69, Reverberation Mapping of the BroadLine Region in Active Galactic Nuclei (San Francisco: ASP), 111

Gaskell, C. M., \& Martin, C. 1996, ApJ, 464, L107

Goad, M., \& Koratkar, A. 1998, ApJ, 495, 718

Guilbert, P. W., \& Rees, M. J. 1988, MNRAS, 233, 475

Inda, M., et al. 1994, ApJ, 420, 143

Kaspi, S., et al. 1996, ApJ, 470, 336

Korista, K. T., et al. 1995, ApJS, 97, 285

Krolik, J. H., Horne, K., Kallman, T. R., Malkan, M. A., Edelson, R. A., \& Kriss, G. A. 1991, ApJ, 371, 541

Leighly, K. M., et al. 1997, ApJ, 483, 767

Lightman, A. P., \& White, T. R. 1988, ApJ, 335, 57

Livio, M., \& Xu, C. 1997, ApJ, 478, L63
Maoz, D., \& Netzer, H. 1989, MNRAS, 236, 21

Maoz, D., et al. 1993, ApJ, 404, 563

Marshall, H. L., et al. 1997, ApJ, 479, 222

Nandra, K., et al. 1998, ApJ, 505, 594

Nichols, J. S., Garhart, M. P., De la Peña, M. D., \& Levay, K. L. 1993, NEWSIPS Information Manual: Low Dispersion Data V1.0, CSC/SD93/6062

O'Brien, P. T., Goad, M. R., \& Gondhalekar, P. M. 1995, MNRAS, 275, 1125

Orr, M. J. L., \& Browne, I. W. A. 1982, MNRAS, 200, 1067

Pérez, E., Penston, M. V., Tadhunter, C., Mediavilla, E., \& Moles, M. 1988, MNRAS, 230, 353

Peterson, B. M. 1993, PASP, 105, 247

Peterson, B. M., et al. 1991, ApJ, 368, 119

. 1992, ApJ, 392, 470 1994, ApJ, 425, 622 1998, PASP, 110, 660

Reichert, G. A., et al. 1994, ApJ, 582, 608

Rodríguez-Pascual, P. M., et al. 1997, ApJS, 110, 9

Rokaki, E., Boisson, C., \& Collin-Souffrin, S. 1992, A\&A, 253, 57

Romanishin, W., et al. 1995, ApJ, 455, 516

Santos-Lleó, M., et al. 1997, ApJS, 112, 271

Stirpe, G. M., et al. 1994, ApJ, 425, 609

Veilleux, S., \& Zheng, W. 1992, ApJ, 377, 89

Wamsteker, W., Wang, T.-G., Schartel, N., \& Vio, R. 1997, MNRAS, 288, 225

Wanders, I. 1997, in ASP Conf. Ser. 113, Emission Lines in Active Galaxies: New Methods and Techniques (San Francisco: ASP), 183

Wanders, I., et al. 1997, ApJS, 113, 69

Warwick, R. S., et al. 1996, ApJ, 470, 349

Weinstein, D., \& Carini, M. 1992, NASA IUE Newsletter, 48, 143

White, R. J., \& Peterson, B. M. 1994, PASP, 106, 879

Zheng, W. 1996, AJ, 111, 1498

Zheng, W., Veilleux, S., \& Grandi, S. A. 1991, ApJ, 381, 411 